\title{
Stable self-similar blowup in the supercritical heat flow of harmonic maps
}

\author{
Pawel Biernat $^{1} \cdot$ Roland Donninger $^{1,2}$. \\ Birgit Schörkhuber ${ }^{2}$
}

Received: 10 February 2017 / Accepted: 25 October 2017 / Published online: 4 November 2017

(C) The Author(s) 2017. This article is an open access publication

\begin{abstract}
We consider the heat flow of corotational harmonic maps from $\mathbb{R}^{3}$ to the threesphere and prove the nonlinear asymptotic stability of a particular self-similar shrinker that is not known in closed form. Our method provides a novel, systematic, robust, and constructive approach to the stability analysis of self-similar blowup in parabolic evolution equations. In particular, we completely avoid using delicate Lyapunov functionals, monotonicity formulas, indirect arguments, or fragile parabolic structure like the maximum principle. As a matter of fact, our approach reduces the nonlinear stability analysis of self-similar shrinkers to the spectral analysis of the associated self-adjoint linearized operators.
\end{abstract}

Mathematics Subject Classification $58 \mathrm{E} 20 \cdot 53 \mathrm{C} 44 \cdot 35 \mathrm{~B} 44 \cdot 35 \mathrm{~B} 35$

Communicated by M. Struwe.

Roland Donninger is supported by the Alexander von Humboldt Foundation via a Sofja Kovalevskaja Award endowed by the German Federal Ministry of Education and Research. Birgit Schörkhuber is supported by the Austrian Science Fund (FWF) via the Hertha Firnberg Program, Project Nr. T 739-N25. Partial support by the Deutsche Forschungsgemeinschaft (DFG), CRC 1060 'The Mathematics of Emergent Effects', is also gratefully acknowledged.

Roland Donninger

roland.donninger@univie.ac.at

Paweł Biernat

pawel.biernat@gmail.com

Birgit Schörkhuber

birgit.schoerkhuber@univie.ac.at

1 Mathematisches Institut, Rheinische Friedrich-Wilhelms-Universität Bonn, Endenicher Allee 60, 53115 Bonn, Germany

2 Fakultät für Mathematik, Universität Wien, Oskar-Morgenstern-Platz 1, 1090 Vienna, Austria 


\section{Introduction}

Let $(M, g)$ and $(N, h)$ be Riemannian manifolds with metrics $g$ and $h$, respectively. A map $U: M \rightarrow N$ is called harmonic if it is a critical point of the functional

$$
\mathcal{S}(U):=\int_{M} g^{j k} \partial_{j} U^{a} \partial_{k} U^{b} h_{a b} \circ U
$$

where we employ Einstein's summation convention throughout. Note that $\mathcal{S}(U)$ is a natural generalization of the Dirichlet energy. The Euler-Lagrange equations associated to $\mathcal{S}$ are

$$
\Delta_{M} U^{a}-g^{j k} \Gamma_{b c}^{a}(U) \partial_{j} U^{b} \partial_{k} U^{c}=0,
$$

where $\Gamma_{b c}^{a}$ are the Christoffel symbols on the target manifold $N$ and

$$
\Delta_{M}=\frac{1}{\sqrt{\operatorname{det} g}} \partial_{j}\left(\sqrt{\operatorname{det} g} g^{j k} \partial_{k}\right)
$$

is the Laplace-Beltrami operator on $M$. The study of harmonic maps is a classical subject in geometric analysis, see e.g. [18,19,26-28,34-37,39]. The basic mathematical questions concern the existence and, ideally, the classification of harmonic maps. A standard tool in this respect is the associated heat flow, i.e., one considers a one-parameter family $\left\{U_{t}: t \geq 0\right\}$ of maps from $M$ to $N$ that evolve according to the heat equation

$$
\partial_{t} U_{t}^{a}=\Delta_{M} U_{t}^{a}-g^{j k} \Gamma_{b c}^{a}\left(U_{t}\right) \partial_{j} U_{t}^{b} \partial_{k} U_{t}^{c} .
$$

The idea then is to take an arbitrary map $U_{0}: M \rightarrow N$ as initial data at $t=0$ and due to the regularizing effects of the heat flow, the solution $U_{t}$ is expected to converge to an equilibrium as $t \rightarrow \infty$. In other words, the heat flow is supposed to deform arbitrary maps into harmonic ones. Indeed, this strategy works well under certain curvature assumptions as is demonstrated in the classical paper [27]. In the general case, however, the flow tends to form singularities (or "blow up") in finite time [1,2,7,8,10,20,21,25,30-33,43,44]. This is a severe obstruction which can only be overcome if one is able to continue the flow past the singularity in a well-defined manner. Such a construction is a challenging endeavor which presupposes a detailed understanding of possible blowup scenarios. Naturally, one is mainly interested in blowup behavior that is stable under small perturbations of the initial data.

In this paper we are interested in singularity formation in the heat flow of harmonic maps $U: \mathbb{S}^{d} \rightarrow \mathbb{S}^{d}$. As it turns out, the blowup is a local phenomenon and the curvature of the base manifold is irrelevant for the asymptotic behavior near the singularity. Consequently, we may equally well consider maps $U: \mathbb{R}^{d} \rightarrow \mathbb{S}^{d}$, cf. [21,38]. Furthermore, we restrict ourselves to the case $d=3$ and assume corotational symmetry. That is to say, we choose standard spherical coordinates $(r, \theta, \varphi)$ on $\mathbb{R}^{3}$, hyperspherical coordinates on $\mathbb{S}^{3}$, and make the ansatz $U(r, \theta, \varphi)=(u(r), \theta, \varphi)$ for the map $U: \mathbb{R}^{3} \rightarrow \mathbb{S}^{3}$. Under this symmetry reduction, the Euler-Lagrange equations associated to the functional $\mathcal{S}$ reduce to a single nonlinear ordinary differential equation for $u$ which reads

$$
u^{\prime \prime}(r)+\frac{2}{r} u^{\prime}(r)-\frac{\sin (2 u(r))}{r^{2}}=0, \quad r \geq 0 .
$$

In order to obtain the associated heat flow, we introduce an artificial time dependence and consider the Cauchy problem for the equation

$$
\partial_{t} u(r, t)-\partial_{r}^{2} u(r, t)-\frac{2}{r} \partial_{r} u(r, t)+\frac{\sin (2 u(r, t))}{r^{2}}=0 .
$$


Our main result shows the existence of a stable self-similar blowup scenario for Eq. (1.1). For the precise formulation we introduce the following function space.

Definition 1.1 Let

$$
\tilde{Y}:=\left\{h \in C_{c}^{\infty}([0, \infty)): h^{(2 k)}(0)=0 \text { for all } k \in \mathbb{N}_{0}\right\}
$$

and set

$$
\|h\|_{Y}:=\left\||\cdot|^{-1} h(|\cdot|)\right\|_{\dot{H}^{2}\left(\mathbb{R}^{5}\right)}+\left\||\cdot|^{-1} h(|\cdot|)\right\|_{\dot{H}^{4}\left(\mathbb{R}^{5}\right)} .
$$

The Banach space $Y$ is defined as the completion of $\tilde{Y}$ with respect to $\|\cdot\|_{Y}$.

Theorem 1.2 There exists an $f_{0} \in C^{\infty}([0, \infty)) \cap Y$ with $f_{0}>0$ on $(0, \infty)$ such that, for any $T_{0}>0$ and $t \in\left[0, T_{0}\right)$,

$$
u_{T_{0}}^{*}(r, t):=f_{0}\left(\frac{r}{\sqrt{T_{0}-t}}\right)
$$

is a solution to Eq. (1.1). Furthermore, there exist $\delta, M, \omega_{0}>0$ such that the following holds. For any $h \in Y$ satisfying $\|h\|_{Y} \leq \frac{\delta}{M^{2}}$, there exists a $T_{h} \in\left[T_{0}-\frac{\delta}{M}, T_{0}+\frac{\delta}{M}\right]$ such that Eq. (1.1) with initial data $u(r, 0)=u_{T_{0}}^{*}(r, 0)+h(r)$ has a unique solution $u_{h}$ that blows up at $t=T_{h}$ and converges to $u_{T_{h}}^{*}$ in the sense that

$$
\frac{\left\|u_{h}(\cdot, t)-u_{T_{h}}^{*}(\cdot, t)\right\|_{Y}}{\left\|u_{T_{h}}^{*}(\cdot, t)\right\|_{Y}} \leq \delta\left(T_{h}-t\right)^{\omega_{0}}
$$

for all $t \in\left[0, T_{h}\right)$. In particular, the class $\left\{u_{T_{0}}^{*}: T_{0}>0\right\}$ of self-similar solutions is nonlinearly asymptotically stable under small perturbations of the initial data.

Some remarks are in order.

- The map $U: \mathbb{R}^{3} \rightarrow \mathbb{S}^{3}$ has values on the sphere and thus, there is no blowup in $L^{\infty}$. However, the self-similar solution $u_{T_{0}}^{*}$ blows up in $Y$. Indeed, a simple scaling argument shows

$$
\left\|u_{T_{0}}^{*}(\cdot, t)\right\|_{Y} \simeq\left(T_{0}-t\right)^{-\frac{5}{4}}
$$

for $t \in\left[0, T_{0}\right)$.

- The blowup profile $f_{0}$ is constructed in the companion paper [3] by a novel computerassisted (but rigorous) method. It is not known in closed form. Furthermore, $f_{0}$ is not the only self-similar profile. In fact, there exist infinitely many self-similar solutions to Eq. (1.1), see [20]. To the knowledge of the authors, Theorem 1.2 is the first result on stable blowup with a nonunique blowup profile that is not known explicitly.

- The norm $\|\cdot\|_{Y}$ might look odd at first glance since it is based on homogeneous Sobolev spaces on $\mathbb{R}^{5}$ whereas Eq. (1.1) is posed on $\mathbb{R}^{3}$. However, if one sets $u(r, t)=r v(r, t)$, Eq. (1.1) transforms into a radial heat equation on $\mathbb{R}^{5}$ for the function $v$. In addition, this transformation regularizes the nonlinearity at the center, see below. In this sense, the effective dimension of the problem is 5 and it is natural to work with radial functions on $\mathbb{R}^{5}$.

- In the formulation of Theorem 1.2 we do not specify the precise solution concept we are using. We will study Eq. (1.1) in similarity coordinates by semigroup theory which yields a canonical notion of strong solution (which is actually called "mild solution" in semigroup theory). Since Eq. (1.1) is parabolic, smoothing effects will kick in immediately and turn strong solutions into classical ones. 
- For obvious reasons, self-similar solutions of the form $f\left(\frac{r}{\sqrt{T_{0}-t}}\right)$ are called shrinkers. Since Eq. (1.1) is not time-reversible, there is another, independent class of self-similar solutions, so-called expanders, which take the form $f\left(\frac{r}{\sqrt{t-T_{0}}}\right)$. The latter have also attracted considerable interest, in particular in connection with the question of unique continuation beyond blowup [2,22,23], but they play no role in the present paper.

\subsection{Related results}

The analysis of harmonic maps is a vast subject that is impossible to review in this paper. We restrict ourselves to a brief discussion of recent blowup results that are directly related to our work and refer the reader to the monographs and survey articles $[18,19,26,28,35,39]$ for the general background.

As already indicated, self-similar solutions for the corotational heat flow of harmonic maps $U: \mathbb{R}^{d} \rightarrow \mathbb{S}^{d}$ for $d \in\{3,4,5,6\}$ are constructed in [20,21]. Expanding self-similar solutions are studied in [23]. For $d \geq 7$, there are no self-similar shrinkers [5] and the blowup is of a more complicated nature [1,4]. The case $d=2$ is of special interest since it is energy-critical and blowup takes place via shrinking of a soliton $[32,33,44]$. The unique continuation beyond blowup is investigated in [2,22]. Needless to say, there are similar results for closely related problems like the Yang-Mills heat flow or the nonlinear heat equation, see the discussion in [17] for a brief overview. Of particular interest in this context is the recent paper [9] which also considers self-similar blowup for a nonlinear heat equation with a blowup profile that is not known in closed form. In contrast to our result, however, the blowup studied in [9] is highly unstable and the necessary spectral properties can be obtained by a perturbative argument.

\subsection{Outline of the proof}

The proof of Theorem 1.2 proceeds by a perturbative construction around the blowup solution $u_{T_{0}}^{*}$. We would like to emphasize that this is a robust approach that uses no structure other than the spectral stability of the self-similar profile $f_{0}$ which is established in [3]. As a consequence, our method provides a universal framework for studying self-similar blowup in general parabolic evolution equations. We briefly outline the main steps.

- We consider Eq. (1.1) with initial data $u(r, 0)=u_{T_{0}}^{*}(r, 0)+h(r)$. By time translation invariance we may assume $T_{0}=1$ and we introduce similarity coordinates $s=-\log (T-$ $t)+\log T, y=\frac{r}{\sqrt{T-t}}$ which go back to [24]. Here, $T>0$ is a free parameter which will be adjusted later. Then we rescale the dependent variable $u$ in a suitable manner to obtain the evolution equation

$$
\partial_{s} \tilde{w}-\partial_{y}^{2} \tilde{w}-\frac{4}{y} \partial_{y} \tilde{w}+\frac{y}{2} \partial_{y} \tilde{w}-\frac{2}{y^{2}} \tilde{w}+\frac{1}{2} \tilde{w}+\frac{\sin (y \tilde{w})}{y^{3}}=0,
$$

where $\tilde{w}=\tilde{w}(y, s)$, with initial data $\tilde{w}(y, 0)=f_{0}(\sqrt{T} y) / y+h(\sqrt{T} y) / y$. This equation has the static solution $\tilde{w}(y, s)=f_{0}(y) / y$. To study its stability, we make the ansatz $\tilde{w}(y, s)=f_{0}(y) / y+w(y, s)$ which leads to an evolution equation of the form

$$
\left\{\begin{array}{l}
\partial_{s} w(\cdot, s)=\hat{\mathcal{L}} w(\cdot, s)+\mathcal{N}(w(\cdot, s)) \\
w(y, 0)=f_{0}(\sqrt{T} y) / y-f_{0}(y) / y+h(\sqrt{T} y) / y
\end{array}\right.
$$

for the perturbation $w$. The linear operator $\hat{\mathcal{L}}$ is given by

$$
\hat{\mathcal{L}}=\partial_{y}^{2}+\frac{4}{y} \partial_{y}-\frac{y}{2} \partial_{y}-\frac{1}{2}-V_{0}(y)
$$


with the potential $V_{0}(y)=\frac{2 \cos \left(2 f_{0}(y)\right)-2}{y^{2}}$ and $\mathcal{N}$ denotes the nonlinear remainder. In the spirit of standard local well-posedness theory we now try to solve Eq. (1.2) by treating the nonlinear terms perturbatively. Consequently, we first have to understand the linearized equation that arises from (1.2) by dropping the nonlinear terms.

- The operator $\hat{\mathcal{L}}$, interpreted as an operator acting on radial functions on $\mathbb{R}^{5}$, has a selfadjoint extension $\mathcal{L}$ on $L_{\sigma}^{2}\left(\mathbb{R}^{5}\right)$ with the weight $\sigma(x)=e^{-|x|^{2} / 4}$. Here we encounter the fundamental problem in studying self-similar blowup for parabolic equations: In order to apply self-adjoint spectral theory, it seems necessary to study the evolution in Sobolev spaces with exponentially decaying weights. This, however, is impossible since one cannot control nonlinear terms in such spaces.

There are (at least) two ways around this issue. First, one can study the evolution in unweighted Sobolev spaces and rely on nonself-adjoint spectral theory. This approach was chosen in [17] for the study of the Yang-Mills heat flow. In this paper we follow a different strategy which is based on the simple observation that in a certain sense the problem splits into a self-adjoint part on a compact domain, where the exponentially decaying weight is irrelevant, and a nonself-adjoint part on an unbounded domain which, however, is easy since the potential term is negligible there. We remark that this is not a new discovery but a well-known phenomenon in parabolic problems, see e.g. $[6,9,29,40]$. Somewhat paradoxically, we can therefore study the linearized evolution on unweighted spaces by using self-adjoint spectral theory in a weighted space.

More precisely, we consider the semigroup $e^{s \mathcal{L}}$ on $L_{\sigma}^{2}\left(\mathbb{R}^{5}\right)$ generated by the self-adjoint operator $\mathcal{L}$. From [3] we know that $\mathcal{L}$ has precisely one nonnegative eigenvalue $\lambda=1$ with eigenfunction $\psi_{1}$. As usual, this instability is related to the freedom in choosing the parameter $T$ in the similarity coordinates. From self-adjoint spectral theory we obtain the weighted decay estimate

$$
\left\|e^{s \mathcal{L}} f\right\|_{L_{\sigma}^{2}} \lesssim e^{-c_{0} s}\|f\|_{L_{\sigma}^{2}}
$$

for some constant $c_{0}>0$, provided $f \perp \psi_{1}$. Similar bounds hold for higher Sobolev spaces with weights. As a matter of fact, also on unweighted homogeneous Sobolev spaces of sufficiently high degree we have decay, but a priori only for the free operator $\mathcal{L}_{0}=\mathcal{L}-V_{0}$. Indeed, an integration by parts shows

$$
\left(\Delta \mathcal{L}_{0} f \mid \Delta f\right)_{L^{2}} \leq-\frac{1}{4}\|f\|_{L^{2}}^{2}
$$

on the unweighted $L^{2}$. Similar bounds hold for higher derivatives. Consequently, by combining the unweighted bounds, the weighted decay, and the smallness of $V_{0}(y)$ for large $y$, we derive the unweighted decay

$$
\left\|e^{s \mathcal{L}} f\right\|_{X} \lesssim e^{-\omega_{0} s}\|f\|_{X}, \quad f \perp \psi_{1}
$$

for some $\omega_{0}>0$, where $X=\dot{H}^{2}\left(\mathbb{R}^{5}\right) \cap \dot{H}^{4}\left(\mathbb{R}^{5}\right)$.

- From now on we follow the argument introduced in our earlier works [11-16] on selfsimilar blowup for wave-type equations. We first show that the nonlinearity is locally Lipschitz on $X$. This is not hard but requires at least some work due to the removable singularity of the nonlinearity at the center. Then we employ Duhamel's principle to rewrite Eq. (1.2) as

$$
\phi(s)=e^{s \mathcal{L}} \mathcal{U}(h, T)+\int_{0}^{s} e^{\left(s-s^{\prime}\right) \mathcal{L}} \mathcal{N}\left(\phi\left(s^{\prime}\right)\right) \mathrm{d} s^{\prime}
$$


where $\phi(s)(y)=w(y, s)$ and $\mathcal{U}(h, T)$ is an abbreviation for the initial data. In general, Eq. (1.3) does not have a global solution due to the unstable eigenvalue $1 \in \sigma(\mathcal{L})$. We deal with this issue by employing the Lyapunov-Perron method. That is to say, we first suppress the instability by subtracting a correction term and instead of Eq. (1.3), we consider the modified equation

$$
\phi(s)=e^{s \mathcal{L}} \mathcal{U}(h, T)+\int_{0}^{s} e^{\left(s-s^{\prime}\right) \mathcal{L}} \mathcal{N}\left(\phi\left(s^{\prime}\right)\right) \mathrm{d} s^{\prime}-e^{s} \mathcal{C}(\phi, \mathcal{U}(h, T))
$$

with

$$
\mathcal{C}(\phi, \mathcal{U}(h, T))=\mathcal{P U}(h, T)+\int_{0}^{\infty} e^{-s^{\prime}} \mathcal{P} \mathcal{N}\left(\phi\left(s^{\prime}\right)\right) \mathrm{d} s^{\prime} .
$$

Here, $\mathcal{P}$ is the orthogonal projection on the unstable subspace $\left\langle\psi_{1}\right\rangle$. By a fixed point argument we show that for any small $h$ and $T$ close to 1, Eq. (1.4) has a global (in $s$ ) solution $\phi_{h, T}$ that decays like the stable linear flow, i.e., $\left\|\phi_{h, T}(s)\right\| \lesssim e^{-\omega_{0} s}$. In the final step we prove that for any small $h$, there exists a $T_{h}$ close to 1 which makes the correction term vanish. In other words, $\phi_{h, T_{h}}$ is a solution to the original equation (1.3).

\section{Preliminary transformations}

The basic evolution equation is

$$
\partial_{t} u(r, t)-\partial_{r}^{2} u(r, t)-\frac{2}{r} \partial_{r} u(r, t)+\frac{\sin (2 u(r, t))}{r^{2}}=0
$$

where $r \geq 0$. For any $T_{0}>0$, we have the self-similar solution

$$
u_{T_{0}}^{*}(r, t)=f_{0}\left(\frac{r}{\sqrt{T_{0}-t}}\right)
$$

with $f_{0}$ constructed in [3]. Our goal is to study the evolution of small initial perturbations of $u_{T_{0}}^{*}$. By time translation invariance, we may restrict ourselves to $T_{0}=1$. Consequently, we consider the Cauchy problem

$$
\left\{\begin{array}{l}
\partial_{t} u(r, t)-\partial_{r}^{2} u(r, t)-\frac{2}{r} \partial_{r} u(r, t)+\frac{\sin (2 u(r, t))}{r^{2}}=0 \\
u(r, 0)=u_{1}^{*}(r, 0)+h(r)=f_{0}(r)+h(r)
\end{array}\right.
$$

where $h$ is a free function. In order to regularize the nonlinearity, it is useful to change variables according to $u(r, t)=r v(r, t)$. This yields

$$
\left\{\begin{array}{l}
\partial_{t} v(r, t)-\partial_{r}^{2} v(r, t)-\frac{4}{r} \partial_{r} v(r, t)-\frac{2}{r^{2}} v(r, t)+\frac{\sin (2 r v(r, t))}{r^{3}}=0 \\
v(r, 0)=f_{0}(r) / r+h(r) / r .
\end{array}\right.
$$

Accordingly, we write $u_{T_{0}}^{*}(r, t)=r v_{T_{0}}^{*}(r, t)$ for the self-similar solution. Now we switch to similarity coordinates $s=-\log (T-t)+\log T, y=\frac{r}{\sqrt{T-t}}$ and define the new dependent variable $\tilde{w}$ by

$$
\tilde{w}(y, s):=\sqrt{T} e^{-s / 2} v\left(\sqrt{T} y e^{-s / 2}, T\left(1-e^{-s}\right)\right),
$$

or, equivalently,

$$
v(r, t)=\frac{1}{\sqrt{T-t}} \tilde{w}\left(\frac{r}{\sqrt{T-t}},-\log (T-t)+\log T\right) .
$$


Here, $T>0$ is a free parameter that will be needed to account for the time translation invariance of the problem which introduces an artificial instability. Eq. (2.3) transforms into

$$
\left\{\begin{array}{l}
\partial_{s} \tilde{w}(y, s)-\partial_{y}^{2} \tilde{w}(y, s)-\frac{4}{y} \partial_{y} \tilde{w}(y, s)+\frac{y}{2} \partial_{y} \tilde{w}(y, s)-\frac{2}{y^{2}} \tilde{w}(y, s)+\frac{1}{2} \tilde{w}(y, s)+\frac{\sin (2 y \tilde{w}(y, s))}{y^{3}}=0 \\
\tilde{w}(y, 0)=f_{0}(\sqrt{T} y) / y+h(\sqrt{T} y) / y .
\end{array}\right.
$$

Observe that the only trace of the parameter $T$ is in the initial data. Furthermore, by construction,

$$
\begin{aligned}
\tilde{w}_{T}^{*}(y, s): & =\sqrt{T} e^{-s / 2} v_{T}^{*}\left(\sqrt{T} y e^{-s / 2}, T\left(1-e^{-s}\right)\right)=\frac{1}{y} u_{T}^{*}\left(\sqrt{T} y e^{-s / 2}, T\left(1-e^{-s}\right)\right) \\
& =f_{0}(y) / y
\end{aligned}
$$

is a static solution to Eq. (2.4). By making the ansatz $\tilde{w}(y, s)=f_{0}(y) / y+w(y, s)$, we rewrite Eq. (2.4) as

$$
\left\{\begin{array}{l}
\partial_{s} w(y, s)=\hat{\mathcal{L}} w(y, s)+\hat{\mathcal{N}}(w(y, s)) \\
w(y, 0)=f_{0}(\sqrt{T} y) / y-f_{0}(y) / y+h(\sqrt{T} y) / y
\end{array}\right.
$$

with the linear operator $\hat{\mathcal{L}}$ defined by

$$
\hat{\mathcal{L}}=\partial_{y}^{2}+\frac{4}{y} \partial_{y}-\frac{y}{2} \partial_{y}-\frac{1}{2}-\frac{2 \cos \left(2 f_{0}(y)\right)-2}{y^{2}}
$$

and the nonlinearity

$$
\hat{\mathcal{N}}(w(y, s))=-\frac{1}{y^{3}}\left[\sin \left(2 f_{0}(y)+2 y w(y, s)\right)-\sin \left(2 f_{0}(y)\right)-2 y \cos \left(2 f_{0}(y)\right) w(y, s)\right] .
$$

\section{The linearized evolution}

In this section we study the linearized equation, i.e., we drop the nonlinearity in Eq. (2.5) and focus on

$$
\partial_{s} w(y, s)=\hat{\mathcal{L}} w(y, s) .
$$

Furthermore, we do not specify the initial data explicitly because their specific form is irrelevant for the linear theory.

Note that the operator $\hat{\mathcal{L}}$ contains the 5-dimensional radial Laplacian and for the rest of this paper we actually find it convenient to switch to 5-dimensional notation. To this end, we define the operator

$$
\Lambda f(x):=\frac{1}{2} x \nabla f(x)+\frac{1}{2} f(x)
$$

acting on functions $f: \mathbb{R}^{5} \rightarrow \mathbb{R}$. In the following, the variable $x$ is used to denote an element of $\mathbb{R}^{5}$. In this spirit we define the potential $V_{0}: \mathbb{R}^{5} \rightarrow \mathbb{R}$ by

$$
V_{0}(x):=-\frac{2 \cos \left(2 f_{0}(|x|)\right)-2}{|x|^{2}}
$$


By [3], $f_{0}$ is odd ${ }^{1}$ and thus, $V_{0} \in C^{\infty}\left(\mathbb{R}^{5}\right)$, see [45]. Now we define a differential operator $\tilde{\mathcal{L}}$ by

$$
\tilde{\mathcal{L}} f:=\Delta f-\Lambda f+V_{0} f
$$

where throughout, $\Delta$ denotes the Laplacian on $\mathbb{R}^{5}$. Then we have

$$
\tilde{\mathcal{L}} f(x)=\tilde{f}^{\prime \prime}(|x|)+\frac{4}{|x|} \tilde{f}^{\prime}(|x|)-\frac{|x|}{2} \tilde{f}^{\prime}(|x|)-\frac{1}{2} \tilde{f}(|x|)+V_{0}(x) \tilde{f}(|x|)
$$

for all radial functions $f: \mathbb{R}^{5} \rightarrow \mathbb{R}$ with $f(x)=\tilde{f}(|x|)$. Consequently, the linearized equation (3.1) can be written as

$$
\partial_{s} \phi(s)=\tilde{\mathcal{L}} \phi(s)
$$

where $\phi(s)(x)=w(|x|, s)$. Formally, the solution of Eq. (3.2) is given by $\phi(s)=e^{s \tilde{\mathcal{L}}} \phi(0)$. In the following, we make this rigorous.

\subsection{Basic semigroup theory}

As usual, for $\Omega \subset \mathbb{R}^{d}$ open and $w: \Omega \rightarrow[0, \infty)$ a weight function, we write

$$
(f \mid g)_{L_{w}^{2}(\Omega)}:=\int_{\Omega} f(x) g(x) w(x) \mathrm{d} x, \quad\|f\|_{L_{w}^{2}(\Omega)}:=\sqrt{(f \mid f)_{L_{w}^{2}(\Omega)}}
$$

and denote by $L_{w}^{2}(\Omega)$ the completion of $C_{c}^{\infty}(\Omega)$ with respect to $\|\cdot\|_{L_{w}^{2}(\Omega)}$.

We promote $\tilde{\mathcal{L}}$ to an unbounded linear operator on the Hilbert space

$$
H:=\left\{f \in L_{\sigma}^{2}\left(\mathbb{R}^{5}\right): f \text { radial }\right\}
$$

with $\sigma(x)=e^{-|x|^{2} / 4}$, by specifying the domain $\mathcal{D}(\tilde{\mathcal{L}}):=\left\{f \in C_{c}^{\infty}\left(\mathbb{R}^{5}\right): f\right.$ radial $\}$.

Proposition 3.1 The operator $\tilde{\mathcal{L}}: \mathcal{D}(\tilde{\mathcal{L}}) \subset H \rightarrow H$ is essentially self-adjoint and the spectrum of its closure $\mathcal{L}$ satisfies $\sigma(\mathcal{L}) \cap[0, \infty)=\{1\}$. The spectral point 1 is a simple eigenvalue and $\mathcal{L}$ generates a strongly continuous one-parameter semigroup $e^{s \mathcal{L}}$ on $H$. The function $\psi_{1}(x):=f_{0}^{\prime}(|x|) /\left\|f_{0}^{\prime}(|\cdot|)\right\|_{L_{\sigma}^{2}\left(\mathbb{R}^{5}\right)}$ is an eigenfunction of $\mathcal{L}$ with eigenvalue 1. Moreover, there exists a constant $c_{0}>0$ such that

$$
\left\|e^{s \mathcal{L}} f\right\|_{L_{\sigma}^{2}\left(\mathbb{R}^{5}\right)} \leq e^{-c_{0} s}\|f\|_{L_{\sigma}^{2}\left(\mathbb{R}^{5}\right)}
$$

for all $f \in H$ satisfying $\left(f \mid \psi_{1}\right)_{L_{\sigma}^{2}\left(\mathbb{R}^{5}\right)}=0$ and all $s \geq 0$.

Proof Via $\tilde{f} \mapsto\left|\mathbb{S}^{4}\right|^{-1 / 2} \tilde{f}(|\cdot|): L_{\rho}^{2}(0, \infty) \rightarrow H$ with the weight $\rho(y)=y^{4} e^{-y^{2} / 4}, \tilde{\mathcal{L}}$ is unitarily equivalent to the Sturm-Liouville operator

$$
\mathcal{T} \tilde{f}(y):=\frac{1}{\rho(y)} \frac{\mathrm{d}}{\mathrm{d} y}\left[\rho(y) \tilde{f}^{\prime}(y)\right]-\frac{1}{2} \tilde{f}(y)-\frac{2 \cos \left(2 f_{0}(y)\right)-2}{y^{2}} \tilde{f}(y)
$$

with domain $\mathcal{D}(\mathcal{T}):=\left\{\tilde{f} \in C_{c}^{\infty}([0, \infty)): \tilde{f}^{(2 k)}(0)=0\right.$ for all $\left.k \in \mathbb{N}_{0}\right\}$. The equation

$$
\frac{1}{\rho(y)} \frac{\mathrm{d}}{\mathrm{d} y}\left[\rho(y) \tilde{f}^{\prime}(y)\right]=0
$$

1 By this we mean that $f_{0}$ can be extended to all of $\mathbb{R}$ as a smooth, odd function. In other words, $f_{0}^{(2 k)}(0)=0$ for all $k \in \mathbb{N}_{0}$. 
has the explicit solution

$$
\tilde{f}_{1}(y)=\int_{1}^{y} \rho(s)^{-1} \mathrm{~d} s=\int_{1}^{y} s^{-4} e^{s^{2} / 4} \mathrm{~d} s .
$$

For $y \in(0,1]$ we have

$$
\left|\tilde{f}_{1}(y)\right|=\int_{y}^{1} s^{-4} e^{s^{2} / 4} \mathrm{~d} s \geq \int_{y}^{1} s^{-4} \mathrm{~d} s=\frac{1}{3} y^{-3}-\frac{1}{3}
$$

and thus, $\tilde{f}_{1} \notin L_{\rho}^{2}(0,1)$. Similarly, for $y \geq 1$,

$$
\tilde{f}_{1}(y)=2 \int_{1}^{y} s^{-5} \partial_{s} e^{s^{2} / 4} \mathrm{~d} s \geq 2 y^{-5}\left(e^{y^{2} / 4}-e^{1 / 4}\right)
$$

which implies $\tilde{f}_{1} \notin L_{\rho}^{2}(1, \infty)$. By the Weyl alternative, the Sturm-Liouville operator defined by (3.3) is in the limit-point case at both endpoints and the Kato-Rellich theorem implies that $\mathcal{T}$ (and hence $\tilde{\mathcal{L}}$ ) is essentially self-adjoint, see e.g. [42].

In fact, by $\tilde{f} \mapsto\left|\mathbb{S}^{4}\right|^{-1 / 2} \tilde{f}(|\cdot|) /|\cdot|: L_{\tilde{\rho}}^{2}(0, \infty) \rightarrow H$ with $\tilde{\rho}(y)=y^{2} e^{-y^{2} / 4}, \mathcal{L}$ is unitarily equivalent to the operator $-\mathcal{A}_{0}$ studied in [3]. Consequently, from [3] we obtain $\sigma(\mathcal{L}) \cap[0, \infty)=\{1\}$ with 1 a simple eigenvalue. The corresponding normalized eigenfunction is given by $\psi_{1}(x)=f_{0}^{\prime}(|x|) /\left\|f_{0}(|\cdot|)\right\|_{L_{\sigma}^{2}\left(\mathbb{R}^{5}\right)}$, see [3]. Since $0 \notin \sigma(\mathcal{L})$, we obtain $-c_{0}:=$ $\sup \sigma(\mathcal{L}) \backslash\{1\}<0$ and the self-adjointness of $\mathcal{L}$ implies the bound

$$
(\mathcal{L} f \mid f)_{L_{\sigma}^{2}\left(\mathbb{R}^{5}\right)} \leq-c_{0}\|f\|_{L_{\sigma}^{2}\left(\mathbb{R}^{5}\right)}^{2}
$$

for all $f \in \mathcal{D}(\mathcal{L})$ with $\left(f \mid \psi_{1}\right)_{L_{\sigma}^{2}\left(\mathbb{R}^{5}\right)}=0$. From this, the stated bound on the semigroup $e^{s \mathcal{L}}$ follows.

\subsection{Estimates in local Sobolev norms}

We upgrade the $L_{\sigma}^{2}$ bound on $e^{s \mathcal{L}}$ to a local $H^{4}$ bound. In the following we use

$$
\|f\|_{G(\mathcal{L})}:=\|\mathcal{L} f\|_{L_{\sigma}^{2}\left(\mathbb{R}^{5}\right)}+\|f\|_{L_{\sigma}^{2}\left(\mathbb{R}^{5}\right)}
$$

for $f \in \mathcal{D}(\mathcal{L})$ to denote the graph norm of $\mathcal{L}$. Furthermore, the letter $C$ (possibly with subscripts to indicate dependencies) denotes a positive constant that might change its value at each occurrence and $c_{0}>0$ is the constant from Proposition 3.1. Finally, for $R>0$ we set

$$
\mathbb{B}_{R}^{5}:=\left\{x \in \mathbb{R}^{5}:|x|<R\right\} .
$$

Lemma 3.2 Let $f \in \mathcal{D}(\mathcal{L})$ and $R \geq 1$. Then $\nabla f, \Delta f \in L^{2}\left(\mathbb{B}_{R}^{5}\right)$ and we have the bound

$$
\|\Delta f\|_{L^{2}\left(\mathbb{B}_{R}^{5}\right)}+\|\nabla f\|_{L^{2}\left(\mathbb{B}_{R}^{5}\right)} \leq C_{R}\|f\|_{G(\mathcal{L})}
$$

for all $R \geq 1$ and all $f \in \mathcal{D}(\mathcal{L})$.

Proof Let $f \in C_{c}^{\infty}\left(\mathbb{R}^{5}\right)$ and $R \geq 1$. An integration by parts yields

$$
(\mathcal{L} f \mid f)_{L_{\sigma}^{2}\left(\mathbb{R}^{5}\right)} \leq-\|\nabla f\|_{L_{\sigma}^{2}\left(\mathbb{R}^{5}\right)}^{2}+C\|f\|_{L_{\sigma}^{2}\left(\mathbb{R}^{5}\right)}^{2}
$$

and we infer

$$
\|\nabla f\|_{L^{2}\left(\mathbb{B}_{R}^{5}\right)} \leq C_{R}\|\nabla f\|_{L_{\sigma}^{2}\left(\mathbb{R}^{5}\right)} \leq C_{R}\|f\|_{G(\mathcal{L})} .
$$


Now let $f \in \mathcal{D}(\mathcal{L})$. Since $C_{c}^{\infty}\left(\mathbb{R}^{5}\right)$ is a core for $\mathcal{L}$, there exists a sequence $\left(f_{n}\right) \subset C_{c}^{\infty}\left(\mathbb{R}^{5}\right)$ such that $f_{n} \rightarrow f$ in the graph norm $\|\cdot\|_{G(\mathcal{L})}$. Consequently, Eq. (3.5) shows that $\left(\partial_{j} f_{n}\right)$ is Cauchy in $L^{2}\left(\mathbb{B}_{R}^{5}\right)$ for any $j \in\{1,2, \ldots, 5\}$. We set $g_{j}:=\lim _{n \rightarrow \infty} \partial_{j} f_{n} \in L^{2}\left(\mathbb{B}_{R}^{5}\right)$. By dominated convergence we infer

$$
\int_{\mathbb{B}_{R}^{5}} g_{j} \varphi=\lim _{n \rightarrow \infty} \int_{\mathbb{B}_{R}^{5}} \partial_{j} f_{n} \varphi=-\lim _{n \rightarrow \infty} \int_{\mathbb{B}_{R}^{5}} f_{n} \partial_{j} \varphi=-\int_{\mathbb{B}_{R}^{5}} f \partial_{j} \varphi
$$

for any $\varphi \in C_{c}^{\infty}\left(\mathbb{B}_{R}^{5}\right)$. Consequently, $\partial_{j} f=g_{j}$ in the weak sense and this shows $\nabla f \in$ $L^{2}\left(\mathbb{B}_{R}^{5}\right)$ with the bound (3.5).

Let $f \in C_{c}^{\infty}\left(\mathbb{R}^{5}\right)$. Then we have

$$
\begin{aligned}
\|\mathcal{L} f\|_{L^{2}\left(\mathbb{B}_{R}^{5}\right)}^{2} & =\left(\Delta f-\Lambda f+V_{0} f \mid \Delta f-\Lambda f+V_{0} f\right)_{L^{2}\left(\mathbb{B}_{R}^{5}\right)} \\
& =\|\Delta f\|_{L^{2}\left(\mathbb{B}_{R}^{5}\right)}^{2}+2\left(\Delta f \mid-\Lambda f+V_{0} f\right)_{L^{2}\left(\mathbb{B}_{R}^{5}\right)}+\left\|-\Lambda f+V_{0} f\right\|_{L^{2}\left(\mathbb{B}_{R}^{5}\right)}^{2}
\end{aligned}
$$

which yields the bound

$$
\begin{aligned}
\|\Delta f\|_{L^{2}\left(\mathbb{B}_{R}^{5}\right)} & \lesssim\|\mathcal{L} f\|_{L^{2}\left(\mathbb{B}_{R}^{5}\right)}+\|\Lambda f\|_{L^{2}\left(\mathbb{B}_{R}^{5}\right)}+\left\|V_{0} f\right\|_{L^{2}\left(\mathbb{B}_{R}^{5}\right)} \\
& \leq C_{R}\|f\|_{G(\mathcal{L})}+C_{R}\|\nabla f\|_{L^{2}\left(\mathbb{B}_{R}^{5}\right)} \\
& \leq C_{R}\|f\|_{G(\mathcal{L})}
\end{aligned}
$$

by Eq. (3.5). Consequently, a density argument as above finishes the proof.

In order to control the full Sobolev norm

$$
\|f\|_{H^{k}\left(\mathbb{B}_{R}^{5}\right)}=\sum_{|\alpha| \leq k}\left\|\partial^{\alpha} f\right\|_{L^{2}\left(\mathbb{B}_{R}^{5}\right)}
$$

for $k=2$, we need two technical results which are completely elementary since we restrict ourselves to radial functions. First, we have a trace lemma.

Lemma 3.3 Let $R \geq 1$. Then we have the bound

$$
\|\nabla f\|_{L^{\infty}\left(\partial \mathbb{B}_{R}^{5}\right)} \leq C_{R}\|\Delta f\|_{L^{2}\left(\mathbb{B}_{R}^{5}\right)}
$$

for all radial $f \in C^{2}\left(\overline{\mathbb{B}_{R}^{5}}\right)$.

Proof By assumption, there exists a function $\tilde{f} \in C^{2}([0, R])$ such that $f(x)=\tilde{f}(|x|)$. The fundamental theorem of calculus yields

$$
\left|R^{4} \tilde{f}^{\prime}(R)\right|=\left|\int_{0}^{R} \partial_{r}\left[r^{4} \tilde{f}^{\prime}(r)\right] \mathrm{d} r\right| \leq C_{R}\left(\int_{0}^{R}\left|\frac{1}{r^{4}} \partial_{r}\left[r^{4} \tilde{f}^{\prime}(r)\right]\right|^{2} r^{8} \mathrm{~d} r\right)^{1 / 2} \leq C_{R}\|\Delta f\|_{L^{2}\left(\mathbb{B}_{R}^{5}\right)}
$$

and thus,

$$
\left|\partial_{j} f(x)\right|=\left|\frac{x_{j}}{|x|} \tilde{f}^{\prime}(|x|)\right| \leq C_{R}\|\Delta f\|_{L^{2}\left(\mathbb{B}_{R}^{5}\right)}
$$

for all $x \in \partial \mathbb{B}_{R}^{5}$ and $j \in\{1,2, \ldots, 5\}$.

Next, by an extension argument and Fourier analysis, we easily get control on mixed derivatives. Here and in the following, $\mathcal{F}$ is the Fourier transform

$$
(\mathcal{F} f)(\xi):=\int_{\mathbb{R}^{5}} e^{-\mathrm{i} \xi x} f(x) \mathrm{d} x .
$$


Lemma 3.4 Let $R \geq 1$. Then we have the bound

$$
\left\|\partial_{j} \partial_{k} f\right\|_{L^{2}\left(\mathbb{B}_{R}^{5}\right)} \leq C_{R}\left(\|\Delta f\|_{L^{2}\left(\mathbb{B}_{R}^{5}\right)}+\|\nabla f\|_{L^{2}\left(\mathbb{B}_{R}^{5}\right)}+\|f\|_{L^{2}\left(\mathbb{B}_{R}^{5}\right)}\right)
$$

for all radial $f \in C^{2}\left(\overline{\mathbb{B}_{R}^{5}}\right)$ and all $j, k \in\{1,2, \ldots, 5\}$.

Proof Let $\tilde{f} \in C^{2}([0, R])$ such that $f(x)=\tilde{f}(|x|)$. We define an extension $\tilde{\mathcal{E}} \tilde{f}$ of $\tilde{f}$ by

$$
\tilde{\mathcal{E}} \tilde{f}(r):=\left\{\begin{array}{ll}
\tilde{f}(r) & \text { for } r \in[0, R] \\
\tilde{f}(2 R-r)-2 \tilde{f}^{\prime}(R)(R-r) & \text { for } r \in(R, 2 R)
\end{array} .\right.
$$

Then we have

$$
\begin{aligned}
\lim _{r \rightarrow R+} \tilde{\mathcal{E}} \tilde{f}(r) & =\tilde{f}(R) \\
\lim _{r \rightarrow R+}(\tilde{\mathcal{E}} \tilde{f})^{\prime}(r) & =\lim _{r \rightarrow R+}\left[-\tilde{f}^{\prime}(2 R-r)+2 \tilde{f}^{\prime}(R)\right]=\tilde{f}^{\prime}(R) \\
\lim _{r \rightarrow R+}(\tilde{\mathcal{E}} \tilde{f})^{\prime \prime}(r) & =\lim _{r \rightarrow R+} \tilde{f}^{\prime \prime}(2 R-r)=\tilde{f}^{\prime \prime}(R)
\end{aligned}
$$

and thus, $\tilde{\mathcal{E}} \tilde{f} \in C^{2}([0,2 R))$. Furthermore,

$$
\begin{aligned}
\int_{R}^{\frac{3}{2} R}|\tilde{\mathcal{E}} \tilde{f}(r)|^{2} r^{4} \mathrm{~d} r & =\int_{\frac{1}{2} R}^{R}|\tilde{\mathcal{E}} \tilde{f}(2 R-r)|^{2}(2 R-r)^{4} \mathrm{~d} r \leq C_{R} \int_{\frac{1}{2} R}^{R}|\tilde{\mathcal{E}} \tilde{f}(2 R-r)|^{2} r^{4} \mathrm{~d} r \\
& \leq C_{R} \int_{\frac{1}{2} R}^{R}|\tilde{f}(r)|^{2} r^{4} \mathrm{~d} r+C_{R}\left|\tilde{f}^{\prime}(R)\right|^{2} .
\end{aligned}
$$

Analogously, we obtain

$$
\left\||\cdot|^{2}(\tilde{\mathcal{E}} \tilde{f})^{(k)}\right\|_{L^{2}\left(R, \frac{3}{2} R\right)} \leq C_{R}\left\||\cdot|^{2} \tilde{f}^{(k)}\right\|_{L^{2}\left(\frac{1}{2} R, R\right)}+C_{R}\left|\tilde{f}^{\prime}(R)\right|
$$

for any $k \in\{0,1,2\}$.

Now let $\chi: \mathbb{R}^{5} \rightarrow[0,1]$ be a smooth cut-off that satisfies $\chi(x)=1$ for $|x| \leq 1$ and $\chi(x)=0$ for $|x| \geq \frac{3}{2}$ and set

$$
\mathcal{E} f(x):=\chi\left(\frac{x}{R}\right) \tilde{\mathcal{E}} \tilde{f}(|x|) .
$$

Then $\mathcal{E} f \in C_{c}^{2}\left(\mathbb{R}^{5}\right)$ with $\operatorname{supp}(\mathcal{E} f) \subset \overline{\mathbb{B}_{\frac{3}{2} R}^{5}}$ and $\mathcal{E} f=f$ on $\mathbb{B}_{R}^{5}$. From Eq. (3.6) and Lemma 3.3 we obtain the bound

$$
\begin{aligned}
\|\Delta \mathcal{E} f\|_{L^{2}\left(\mathbb{R}^{5}\right)} & \simeq\|\Delta \mathcal{E} f\|_{L^{2}\left(\mathbb{B}_{R}^{5}\right)}+\|\Delta \mathcal{E} f\|_{L^{2}\left(\mathbb{B}_{\frac{3}{2} R}^{5} \backslash \mathbb{B}_{R}^{5}\right)} \\
& \leq C_{R}\left(\|\Delta f\|_{L^{2}\left(\mathbb{B}_{R}^{5}\right)}+\|\nabla f\|_{L^{2}\left(\mathbb{B}_{R}^{5}\right)}+\|f\|_{L^{2}\left(\mathbb{B}_{R}^{5}\right)}\right) .
\end{aligned}
$$

Consequently, the estimate

$$
\left\|\partial_{j} \partial_{k} f\right\|_{L^{2}\left(\mathbb{B}_{R}^{5}\right)} \leq\left\|\partial_{j} \partial_{k} \mathcal{E} f\right\|_{L^{2}\left(\mathbb{R}^{5}\right)} \lesssim\left\||\cdot|^{2} \mathcal{F} \mathcal{E} f\right\|_{L^{2}\left(\mathbb{R}^{5}\right)} \simeq\|\Delta \mathcal{E} f\|_{L^{2}\left(\mathbb{R}^{5}\right)}
$$

finishes the proof.

Now we can control the linear evolution on the Sobolev space $H^{2}\left(\mathbb{B}_{R}^{5}\right)$. 
Corollary 3.5 Let $f \in \mathcal{D}(\mathcal{L})$ and $R \geq 1$. Then $e^{s \mathcal{L}} f \in H^{2}\left(\mathbb{B}_{R}^{5}\right)$ for all $s \geq 0$ and we have the bound

$$
\left\|e^{s \mathcal{L}} f\right\|_{H^{2}\left(\mathbb{B}_{R}^{5}\right)} \leq C_{R} e^{-c_{0} s}\|f\|_{G(\mathcal{L})}
$$

for all $s \geq 0$ and all $f \in \mathcal{D}(\mathcal{L})$ satisfying $\left(f \mid \psi_{1}\right)_{L_{\sigma}^{2}\left(\mathbb{R}^{5}\right)}=0$.

Proof By Lemma 3.4 it suffices to control $\nabla e^{s \mathcal{L}} f$ and $\Delta e^{s \mathcal{L}} f$. Since $\mathcal{D}(\mathcal{L})$ is invariant under $e^{s \mathcal{L}}$, Lemma 3.2 implies $\nabla e^{s \mathcal{L}} f, \Delta e^{s \mathcal{L}} f \in L^{2}\left(\mathbb{B}_{R}^{5}\right)$. Consequently, from Lemma 3.2 and Proposition 3.1 we infer

$$
\begin{aligned}
\left\|\Delta e^{s \mathcal{L}} f\right\|_{L^{2}\left(\mathbb{B}_{R}^{5}\right)}+\left\|\nabla e^{s \mathcal{L}} f\right\|_{L^{2}\left(\mathbb{B}_{R}^{5}\right)} & \leq C_{R}\left(\left\|\mathcal{L} e^{s \mathcal{L}} f\right\|_{L_{\sigma}^{2}\left(\mathbb{R}^{5}\right)}+\left\|e^{s \mathcal{L}} f\right\|_{L_{\sigma}^{2}\left(\mathbb{R}^{5}\right)}\right) \\
& =C_{R}\left(\left\|e^{s \mathcal{L}} \mathcal{L} f\right\|_{L_{\sigma}^{2}\left(\mathbb{R}^{5}\right)}+\left\|e^{s \mathcal{L}} f\right\|_{L_{\sigma}^{2}\left(\mathbb{R}^{5}\right)}\right) \\
& \leq C_{R} e^{-c_{0} s}\|f\|_{G(\mathcal{L})}
\end{aligned}
$$

since

$$
\left(\mathcal{L} f \mid \psi_{1}\right)_{L_{\sigma}^{2}\left(\mathbb{R}^{5}\right)}=\left(f \mid \mathcal{L} \psi_{1}\right)_{L_{\sigma}^{2}\left(\mathbb{R}^{5}\right)}=\left(f \mid \psi_{1}\right)_{L_{\sigma}^{2}\left(\mathbb{R}^{5}\right)}=0 .
$$

Next, we improve the above by two derivatives.

Lemma 3.6 Let $f \in \mathcal{D}\left(\mathcal{L}^{2}\right)$ and $R \geq 1$. Then $\nabla \Delta f, \Delta^{2} f \in L^{2}\left(\mathbb{B}_{R}^{5}\right)$ and we have the bound

$$
\left\|\Delta^{2} f\right\|_{L^{2}\left(\mathbb{B}_{R}^{5}\right)}+\|\nabla \Delta f\|_{L^{2}\left(\mathbb{B}_{R}^{5}\right)} \leq C_{R}\|f\|_{G\left(\mathcal{L}^{2}\right)}
$$

for all $R \geq 1$ and all $f \in \mathcal{D}\left(\mathcal{L}^{2}\right)$.

Proof Let $f \in C_{c}^{\infty}\left(\mathbb{R}^{5}\right)$ and $R \geq 1$. From Lemma 3.2 we have the bound

$$
\|\nabla \mathcal{L} f\|_{L^{2}\left(\mathbb{B}_{R}^{5}\right)} \leq C_{R}\|f\|_{G\left(\mathcal{L}^{2}\right)} .
$$

Expanding the square yields

$$
\begin{aligned}
\|\nabla \mathcal{L} f\|_{L^{2}\left(\mathbb{B}_{R}^{5}\right)}^{2}= & \left\|\nabla\left(\Delta-\Lambda+V_{0}\right) f\right\|_{L^{2}\left(\mathbb{B}_{R}^{5}\right)}^{2} \\
= & \|\nabla \Delta f\|_{L^{2}\left(\mathbb{B}_{R}^{5}\right)}^{2}+2\left(\nabla \Delta f \mid \nabla\left(-\Lambda+V_{0}\right) f\right)_{L^{2}\left(\mathbb{B}_{R}^{5}\right)} \\
& +\left\|\nabla\left(-\Lambda+V_{0}\right) f\right\|_{L^{2}\left(\mathbb{B}_{R}^{5}\right)}^{2}
\end{aligned}
$$

and thus,

$$
\|\nabla \Delta f\|_{L^{2}\left(\mathbb{B}_{R}^{5}\right)} \lesssim\|\nabla \mathcal{L} f\|_{L^{2}\left(\mathbb{B}_{R}^{5}\right)}+\|f\|_{H^{2}\left(\mathbb{B}_{R}^{5}\right)} \leq C_{R}\|f\|_{G\left(\mathcal{L}^{2}\right)}
$$

by Lemmas 3.2 and 3.4 .

For $\Delta^{2} f$ we expand $\|\Delta \mathcal{L} f\|_{L^{2}\left(\mathbb{B}_{R}^{5}\right)}^{2}$ and use Lemma 3.2 together with the bound on $\nabla \Delta f$ to obtain

$$
\left\|\Delta^{2} f\right\|_{L^{2}\left(\mathbb{B}_{R}^{5}\right)} \lesssim\|\Delta \mathcal{L} f\|_{L^{2}\left(\mathbb{B}_{R}^{5}\right)}^{2}+\|\nabla \Delta f\|_{L^{2}\left(\mathbb{B}_{R}^{5}\right)}^{2}+C_{R}\|f\|_{G(\mathcal{L})}^{2} \leq C_{R}\|f\|_{G\left(\mathcal{L}^{2}\right)}^{2} .
$$


Corollary 3.7 Let $f \in \mathcal{D}\left(\mathcal{L}^{2}\right)$ and $R \geq 1$. Then $e^{s \mathcal{L}} f \in H^{4}\left(\mathbb{B}_{R}^{5}\right)$ for all $s \geq 0$ and we have the bound

$$
\left\|e^{s \mathcal{L}} f\right\|_{H^{4}\left(\mathbb{B}_{R}^{5}\right)} \leq C_{R} e^{-c_{0} s}\|f\|_{G\left(\mathcal{L}^{2}\right)}
$$

for all $s \geq 0$ and all $f \in \mathcal{D}\left(\mathcal{L}^{2}\right)$ satisfying $\left(f \mid \psi_{1}\right)_{L_{\sigma}^{2}\left(\mathbb{R}^{5}\right)}=0$.

Proof It suffices to note that $\mathcal{D}\left(\mathcal{L}^{2}\right)$ is invariant under $e^{s \mathcal{L}}$ so that Lemma 3.6 can be applied to $e^{s \mathcal{L}} f$. Proposition 3.1 and Lemma 3.4 then yields the statement.

\subsection{Estimates in unweighted global Sobolev norms}

Next, we prove bounds in $\dot{H}^{2}\left(\mathbb{R}^{5}\right)$ and $\dot{H}^{4}\left(\mathbb{R}^{5}\right)$. The intersection $\dot{H}^{2}\left(\mathbb{R}^{5}\right) \cap \dot{H}^{4}\left(\mathbb{R}^{5}\right)$ will be our main space where we study the evolution. First, we have to ensure that unweighted Sobolev spaces are invariant under $e^{s \mathcal{L}}$.

Lemma 3.8 Let $k \in \mathbb{N}_{0}$ and $f \in H^{k}\left(\mathbb{R}^{5}\right)$. Then we have $e^{s \mathcal{L}} f \in H^{k}\left(\mathbb{R}^{5}\right)$ for all $s \geq 0$ and $e^{s \mathcal{L}}$ is a strongly continuous semigroup on $H^{k}\left(\mathbb{R}^{5}\right)$.

Proof We denote by $\mathcal{L}_{0}=\mathcal{L}-V_{0}+\frac{1}{2}$ the principal part of $\mathcal{L}$. The operator $\mathcal{L}_{0}: \mathcal{D}(\mathcal{L}) \subset$ $L_{\sigma}^{2}\left(\mathbb{R}^{5}\right) \rightarrow L_{\sigma}^{2}\left(\mathbb{R}^{5}\right)$ is self-adjoint and it generates the semigroup $e^{s \mathcal{L}_{0}}$. As a matter of fact, $e^{s \mathcal{L}_{0}}$ can be given explicitly and we have

$$
\left[e^{s \mathcal{L}_{0}} f\right](x)=\left(K_{s} * f\right)\left(e^{-s / 2} x\right)
$$

where

$$
K_{S}(x)=[\pi \alpha(s)]^{-5 / 2} e^{-|x|^{2} / \alpha(s)}, \quad \alpha(s)=4\left(1-e^{-s}\right) .
$$

This is easily verified by an explicit computation. Since $K_{s} \in L^{1}\left(\mathbb{R}^{5}\right)$ for any $s>0$, dominated convergence and Young's inequality immediately imply the invariance of $H^{k}\left(\mathbb{R}^{5}\right)$ under $e^{s \mathcal{L}_{0}}$. By rescaling we infer

$$
\begin{aligned}
e^{s \mathcal{L}_{0}} f(x)-f(x) & =[\pi \alpha(s)]^{-\frac{5}{2}} \int_{\mathbb{R}^{5}} e^{-\left|x^{\prime}\right|^{2} / \alpha(s)}\left[f\left(e^{-s / 2} x-x^{\prime}\right)-f(x)\right] \mathrm{d} x^{\prime} \\
& =\pi^{-\frac{5}{2}} \int_{\mathbb{R}^{5}}\left[f\left(e^{-s / 2} x-\alpha(s)^{\frac{1}{2}} x^{\prime}\right)-f(x)\right] e^{-\left|x^{\prime}\right|^{2}} \mathrm{~d} x^{\prime}
\end{aligned}
$$

and Minkowski's inequality yields

$$
\left\|e^{s \mathcal{L}_{0}} f-f\right\|_{L^{2}\left(\mathbb{R}^{5}\right)} \lesssim \int_{\mathbb{R}^{5}}\left\|f\left(e^{-s / 2}(\cdot)-\alpha(s)^{\frac{1}{2}} x^{\prime}\right)-f\right\|_{L^{2}\left(\mathbb{R}^{5}\right)} e^{-\left|x^{\prime}\right|^{2}} \mathrm{~d} x^{\prime} .
$$

Since scaling and translation are continuous operations on $L^{2}\left(\mathbb{R}^{5}\right)$, we infer

$$
\left\|f\left(e^{-s / 2}(\cdot)-\alpha(s)^{\frac{1}{2}} x^{\prime}\right)-f\right\|_{L^{2}\left(\mathbb{R}^{5}\right)} \rightarrow 0
$$

as $s \rightarrow 0+$ for any fixed $x^{\prime} \in \mathbb{R}^{5}$. Consequently, by dominated convergence, we obtain

$$
\left\|e^{s \mathcal{L}_{0}} f-f\right\|_{L^{2}\left(\mathbb{R}^{5}\right)} \rightarrow 0
$$

as $s \rightarrow 0+$. The same argument yields $\left\|e^{s \mathcal{L}_{0}} f-f\right\|_{H^{k}\left(\mathbb{R}^{5}\right)} \rightarrow 0$ as $s \rightarrow 0+$. We conclude that $e^{s \mathcal{L}_{0}}$ is strongly continuous on $H^{k}\left(\mathbb{R}^{5}\right)$. Evidently, the map $f \mapsto V_{0} f$ is bounded on $H^{k}\left(\mathbb{R}^{5}\right)$ and thus, by the bounded perturbation theorem, $e^{s \mathcal{L}}$ is a strongly continuous semigroup on $H^{k}\left(\mathbb{R}^{5}\right)$. 
Lemma 3.9 Let $f \in \mathcal{D}(\mathcal{L}) \cap \dot{H}^{2}\left(\mathbb{R}^{5}\right)$. Then $e^{s \mathcal{L}} f \in \dot{H}^{2}\left(\mathbb{R}^{5}\right)$ for all $s \geq 0$ and there exists a constant $c_{1}>0$ such that

$$
\left\|e^{s \mathcal{L}} f\right\|_{\dot{H}^{2}\left(\mathbb{R}^{5}\right)} \lesssim e^{-c_{1} s}\left(\|f\|_{G(\mathcal{L})}+\|f\|_{\dot{H}^{2}\left(\mathbb{R}^{5}\right)}\right)
$$

for all $s \geq 0$ and all $f \in \mathcal{D}(\mathcal{L}) \cap \dot{H}^{2}\left(\mathbb{R}^{5}\right)$ satisfying $\left(f \mid \psi_{1}\right)_{L_{\sigma}^{2}\left(\mathbb{R}^{5}\right)}=0$.

Proof Let $f \in C_{c}^{\infty}\left(\mathbb{R}^{5}\right)$ and note the commutator relation $[\Delta, \Lambda] f=\Delta f$ which yields

$$
\Delta \mathcal{L} f=\Delta^{2} f-\Delta \Lambda f+\Delta\left(V_{0} f\right)=\Delta^{2} f-\Lambda \Delta f-\Delta f+\Delta\left(V_{0} f\right) .
$$

Consequently, with $(-\Lambda \Delta f \mid \Delta f)_{L^{2}\left(\mathbb{R}^{5}\right)}=\frac{3}{4}\|\Delta f\|_{L^{2}\left(\mathbb{R}^{5}\right)}^{2}$ we obtain

$$
\begin{aligned}
(\Delta \mathcal{L} f \mid \Delta f)_{L^{2}\left(\mathbb{R}^{5}\right)}= & -\|\nabla \Delta f\|_{L^{2}\left(\mathbb{R}^{5}\right)}^{2} \\
& -(\Lambda \Delta f \mid \Delta f)_{L^{2}\left(\mathbb{R}^{5}\right)}-\|\Delta f\|_{L^{2}\left(\mathbb{R}^{5}\right)}^{2}+\left(\Delta\left(V_{0} f\right) \mid \Delta f\right)_{L^{2}\left(\mathbb{R}^{5}\right)} \\
\leq & -\|\nabla \Delta f\|_{L^{2}\left(\mathbb{R}^{5}\right)}^{2}-\frac{1}{4}\|\Delta f\|_{L^{2}\left(\mathbb{R}^{5}\right)}^{2}+\left(\Delta\left(V_{0} f\right) \mid \Delta f\right)_{L^{2}\left(\mathbb{R}^{5}\right)}
\end{aligned}
$$

Now we claim the estimate

$$
\left|\left(\Delta\left(V_{0} f\right) \mid \Delta f\right)_{L^{2}\left(\mathbb{R}^{5}\right)}\right| \leq C_{R}\|f\|_{G(\mathcal{L})}^{2}+\frac{C}{R^{2}}\|\Delta f\|_{L^{2}\left(\mathbb{R}^{5}\right)}^{2}
$$

for all $R \geq 1$. To prove this, we note that $\Delta\left(V_{0} f\right)=\Delta V_{0} f+2 \nabla V_{0} \nabla f+V_{0} \Delta f$ and estimate each of these terms individually. Clearly,

$$
\begin{aligned}
\left|\left(V_{0} \Delta f \mid \Delta f\right)_{L^{2}\left(\mathbb{R}^{5}\right)}\right| & \lesssim\left\|\left|V_{0}\right|^{\frac{1}{2}} \Delta f\right\|_{L^{2}\left(\mathbb{R}^{5}\right)}^{2}=\left\|\left|V_{0}\right|^{\frac{1}{2}} \Delta f\right\|_{L^{2}\left(\mathbb{B}_{R}^{5}\right)}^{2}+\left\|\left|V_{0}\right|^{\frac{1}{2}} \Delta f\right\|_{L^{2}\left(\mathbb{R}^{5} \backslash \mathbb{B}_{R}^{5}\right)}^{2} \\
& \leq C_{R}\|f\|_{G(\mathcal{L})}^{2}+\frac{C}{R^{2}}\|\Delta f\|_{L^{2}\left(\mathbb{R}^{5}\right)}^{2}
\end{aligned}
$$

where we have used Lemma 3.2 and the decay $\left|V_{0}(x)\right| \lesssim\langle x\rangle^{-2}$. Next,

$$
\left|\left(\nabla V_{0} \nabla f \mid \Delta f\right)_{L^{2}\left(\mathbb{R}^{5}\right)}\right| \lesssim\left\|\left|\nabla V_{0}\right|^{\frac{2}{3}} \nabla f\right\|_{L^{2}\left(\mathbb{R}^{5}\right)}^{2}+\left\|\left|\nabla V_{0}\right|^{\frac{1}{3}} \Delta f\right\|_{L^{2}\left(\mathbb{R}^{5}\right)}^{2} .
$$

Thanks to the decay $\left|\nabla V_{0}(x)\right| \lesssim\langle x\rangle^{-3}$, the last term can be estimated as before. For the first term we use the decay of $\nabla V_{0}$, Lemma 3.2, and Hardy's inequality to estimate

$$
\begin{aligned}
\left\|\left|\nabla V_{0}\right|^{\frac{2}{3}} \nabla f\right\|_{L^{2}\left(\mathbb{R}^{5}\right)} & \lesssim\left\|\langle\cdot\rangle^{-2} \nabla f\right\|_{L^{2}\left(\mathbb{R}^{5}\right)} \simeq\|\nabla f\|_{L^{2}\left(\mathbb{B}_{R}^{5}\right)}+\left\||\cdot|^{-2} \nabla f\right\|_{L^{2}\left(\mathbb{R}^{5} \backslash \mathbb{B}_{R}^{5}\right)} \\
& \leq C_{R}\|f\|_{G(\mathcal{L})}+\frac{C}{R}\left\||\cdot|^{-1} \nabla f\right\|_{L^{2}\left(\mathbb{R}^{5} \backslash \mathbb{B}_{R}^{5}\right)} \\
& \leq C_{R}\|f\|_{G(\mathcal{L})}+\frac{C}{R}\left\||\cdot|^{-1} \nabla f\right\|_{L^{2}\left(\mathbb{R}^{5}\right)} \\
& \leq C_{R}\|f\|_{G(\mathcal{L})}+\frac{C}{R}\|\Delta f\|_{L^{2}\left(\mathbb{R}^{5}\right)} .
\end{aligned}
$$

In view of the decay $\left|\Delta V_{0}(x)\right| \lesssim\langle x\rangle^{-4}$, the term $\left(\Delta V_{0} f \mid \Delta f\right)_{L^{2}\left(\mathbb{R}^{5}\right)}$ can be estimated analogously. This proves Eq. (3.8).

Having Eq. (3.8) at our disposal, we obtain from Eq. (3.7) the bound

$$
(\Delta \mathcal{L} f \mid \Delta f)_{L^{2}\left(\mathbb{R}^{5}\right)} \leq\left(-\frac{1}{4}+\frac{C}{R^{2}}\right)\|\Delta f\|_{L^{2}\left(\mathbb{R}^{5}\right)}^{2}+C_{R}\|f\|_{G(\mathcal{L})}^{2} .
$$


By approximation, Eq. (3.9) extends to all $f \in \mathcal{D}(\mathcal{L})$ satisfying $\mathcal{L} f, f \in H^{2}\left(\mathbb{R}^{5}\right)$. From Lemma 3.8 we know that $\mathcal{L} e^{s \mathcal{L}} f, e^{s \mathcal{L}} f \in H^{2}\left(\mathbb{R}^{5}\right)$ and Eq. (3.9) yields

$$
\begin{aligned}
\frac{1}{2} \partial_{s}\left\|\Delta e^{s \mathcal{L}} f\right\|_{L^{2}\left(\mathbb{R}^{5}\right)}^{2} & =\left(\partial_{s} \Delta e^{s \mathcal{L}} f \mid \Delta e^{s \mathcal{L}} f\right)_{L^{2}\left(\mathbb{R}^{5}\right)}=\left(\Delta \mathcal{L} e^{s \mathcal{L}} f \mid \Delta e^{s \mathcal{L}} f\right)_{L^{2}\left(\mathbb{R}^{5}\right)} \\
& \leq\left(-\frac{1}{4}+\frac{C}{R^{2}}\right)\left\|\Delta e^{s \mathcal{L}} f\right\|_{L^{2}\left(\mathbb{R}^{5}\right)}^{2}+C_{R}\left\|e^{s \mathcal{L}} f\right\|_{G(\mathcal{L})}^{2} \\
& \leq-\frac{1}{8}\left\|\Delta e^{s \mathcal{L}} f\right\|_{L^{2}\left(\mathbb{R}^{5}\right)}^{2}+C_{R} e^{-2 c_{0} s}\|f\|_{G(\mathcal{L})}^{2}
\end{aligned}
$$

by choosing $R \geq 1$ sufficiently large. From now on $R$ is fixed and hence, $C_{R}=C$. Upon setting $c_{1}=\frac{1}{2} \min \left\{c_{0}, \frac{1}{8}\right\}>0$, we infer

$$
\frac{1}{2} \partial_{s}\left\|\Delta e^{s \mathcal{L}} f\right\|_{L^{2}\left(\mathbb{R}^{5}\right)}^{2} \leq-2 c_{1}\left\|\Delta e^{s \mathcal{L}} f\right\|_{L^{2}\left(\mathbb{R}^{5}\right)}^{2}+C e^{-4 c_{1} s}\|f\|_{G(\mathcal{L})}^{2}
$$

and this inequality may be rewritten as

$$
\frac{1}{2} \partial_{s}\left[e^{4 c_{1} s}\left\|\Delta e^{s \mathcal{L}} f\right\|_{L^{2}\left(\mathbb{R}^{5}\right)}^{2}\right] \leq C\|f\|_{G(\mathcal{L})}^{2} .
$$

Consequently, integration yields the bound

$$
\begin{aligned}
\left\|\Delta e^{s \mathcal{L}} f\right\|_{L^{2}\left(\mathbb{R}^{5}\right)}^{2} & \lesssim\langle s\rangle e^{-4 c_{1} s}\left(\|f\|_{G(\mathcal{L})}^{2}+\|f\|_{\dot{H}^{2}\left(\mathbb{R}^{5}\right)}^{2}\right) \\
& \lesssim e^{-2 c_{1} s}\left(\|f\|_{G(\mathcal{L})}^{2}+\|f\|_{\dot{H}^{2}\left(\mathbb{R}^{5}\right)}^{2}\right) .
\end{aligned}
$$

By a density argument, this bound holds for all $f \in \mathcal{D}(\mathcal{L}) \cap \dot{H}^{2}\left(\mathbb{R}^{5}\right)$.

It is now straightforward to upgrade to $\dot{H}^{4}$.

Lemma 3.10 Let $f \in \mathcal{D}\left(\mathcal{L}^{2}\right) \cap \dot{H}^{2}\left(\mathbb{R}^{5}\right) \cap \dot{H}^{4}\left(\mathbb{R}^{5}\right)$. Then $e^{s \mathcal{L}} f \in \dot{H}^{4}\left(\mathbb{R}^{5}\right)$ for all $s \geq 0$ and there exists a constant $c_{1}>0$ such that

$$
\left\|e^{s \mathcal{L}} f\right\|_{\dot{H}^{4}\left(\mathbb{R}^{5}\right)} \lesssim e^{-c_{1} s}\left(\|f\|_{G\left(\mathcal{L}^{2}\right)}+\|f\|_{\dot{H}^{2}\left(\mathbb{R}^{5}\right)}+\|f\|_{\dot{H}^{4}\left(\mathbb{R}^{5}\right)}\right)
$$

for all $s \geq 0$ and all $f \in \mathcal{D}\left(\mathcal{L}^{2}\right) \cap \dot{H}^{2}\left(\mathbb{R}^{5}\right) \cap \dot{H}^{4}\left(\mathbb{R}^{5}\right)$ satisfying $\left(f \mid \psi_{1}\right)_{L_{\sigma}^{2}\left(\mathbb{R}^{5}\right)}=0$.

Proof Let $f \in C_{c}^{\infty}\left(\mathbb{R}^{5}\right)$. By applying the commutator relation $[\Delta, \Lambda] f=\Delta f$ twice, we obtain the estimate

$$
\left(\Delta^{2} \mathcal{L} f \mid \Delta^{2} f\right)_{L^{2}\left(\mathbb{R}^{5}\right)} \leq-\frac{5}{4}\left\|\Delta^{2} f\right\|_{L^{2}\left(\mathbb{R}^{5}\right)}^{2}+\left(\Delta^{2}\left(V_{0} f\right) \mid \Delta^{2} f\right)_{L^{2}\left(\mathbb{R}^{5}\right)},
$$

cf. Eq. (3.7). Consequently, it suffices to follow the logic in the proof of Lemma 3.9 and apply Lemma 3.6.

\subsection{Control of the linearized flow}

Finally, we arrive at the main result on the linearized flow. First, we define the main Sobolev space we will be working with and prove an elementary embedding result.

Definition 3.11 The Banach space $X$ is defined as the completion of all radial functions in $C_{c}^{\infty}\left(\mathbb{R}^{5}\right)$ with respect to the norm

$$
\|f\|_{X}=\|f\|_{\dot{H}^{2}\left(\mathbb{R}^{5}\right) \cap \dot{H}^{4}\left(\mathbb{R}^{5}\right)}=\|\Delta f\|_{L^{2}\left(\mathbb{R}^{5}\right)}+\left\|\Delta^{2} f\right\|_{L^{2}\left(\mathbb{R}^{5}\right)} .
$$


Lemma 3.12 Let $s \in\left[0, \frac{3}{2}\right)$. Then we have the bound

$$
\left\||\nabla|^{s} f\right\|_{L^{\infty}\left(\mathbb{R}^{5}\right)} \lesssim\|f\|_{X}
$$

for all $f \in C_{c}^{\infty}\left(\mathbb{R}^{5}\right)$.

Proof We readily estimate

$$
\begin{aligned}
\left\||\nabla|^{s} f\right\|_{L^{\infty}\left(\mathbb{R}^{5}\right)} & \lesssim\left\||\cdot|^{s} \mathcal{F} f\right\|_{L^{1}\left(\mathbb{R}^{5}\right)} \simeq\left\||\cdot|^{s} \mathcal{F} f\right\|_{L^{1}\left(\mathbb{B}^{5}\right)}+\left\||\cdot|{ }^{s} \mathcal{F} f\right\|_{L^{1}\left(\mathbb{R}^{5} \backslash \mathbb{B}^{5}\right)} \\
& \lesssim\left\||\cdot|^{-2}\right\|_{L^{2}\left(\mathbb{B}^{5}\right)}\left\||\cdot|^{2} \mathcal{F} f\right\|_{L^{2}\left(\mathbb{R}^{5}\right)}+\left\||\cdot|^{-4+s}\right\|_{L^{2}\left(\mathbb{R}^{5} \backslash \mathbb{B}^{5}\right)}\left\||\cdot|^{4} \mathcal{F} f\right\|_{L^{2}\left(\mathbb{R}^{5}\right)} \\
& \lesssim\|f\|_{\dot{H}^{2}\left(\mathbb{R}^{5}\right)}+\|f\|_{\dot{H}^{4}\left(\mathbb{R}^{5}\right)}
\end{aligned}
$$

Now we can prove the following simple but useful embedding theorem.

Lemma 3.13 We have the continuous embeddings

$$
H_{\mathrm{rad}}^{4}\left(\mathbb{R}^{5}\right) \hookrightarrow X \hookrightarrow C^{1}\left(\mathbb{R}^{5}\right) \cap W^{1, \infty}\left(\mathbb{R}^{5}\right)
$$

where $H_{\mathrm{rad}}^{4}\left(\mathbb{R}^{5}\right)=\left\{f \in H^{4}\left(\mathbb{R}^{5}\right): f\right.$ radial $\}$.

Proof Let $f \in H_{\mathrm{rad}}^{4}\left(\mathbb{R}^{5}\right)$. Then there exists a sequence $\left(f_{n}\right)_{n \in \mathbb{N}} \subset C_{c}^{\infty}\left(\mathbb{R}^{5}\right)$ of radial functions such that $f_{n} \rightarrow f$ with respect to $\|\cdot\|_{H^{4}\left(\mathbb{R}^{5}\right)}$. This implies that $\left(f_{n}\right)_{n \in \mathbb{N}}$ is Cauchy with respect to $\|\cdot\|_{X}$ and thus, there exists a limiting element $\hat{f} \in X$ such that $f_{n} \rightarrow \hat{f}$ in $X$. We define a map $\iota: H_{\text {rad }}^{4}\left(\mathbb{R}^{5}\right) \rightarrow X$ by setting $\iota(f):=\hat{f}$. Obviously, $\iota$ is linear. We claim that $\iota$ is injective. Indeed, if $\iota(f)=0$, there exists a sequence $\left(f_{n}\right)_{n \in \mathbb{N}} \subset C_{c}^{\infty}\left(\mathbb{R}^{5}\right)$ that converges to $f$ in $H_{\text {rad }}^{4}\left(\mathbb{R}^{5}\right)$ and to 0 in $X$. By Lemma 3.12 we see that $\lim _{n \rightarrow \infty}\left\|f_{n}\right\|_{L^{\infty}\left(\mathbb{R}^{5}\right)}=0$. In particular, $f_{n} \rightarrow 0$ in $L^{2}\left(\mathbb{R}^{5}\right)$. On the other hand, $f_{n} \rightarrow f$ in $H_{\text {rad }}^{4}\left(\mathbb{R}^{5}\right)$ implies $f_{n} \rightarrow f$ in $L^{2}\left(\mathbb{R}^{5}\right)$ and the uniqueness of weak limits shows that $f=0$. Clearly, we have $\|\iota(f)\|_{X} \lesssim\|f\|_{H^{4}\left(\mathbb{R}^{5}\right)}$ and thus, $\iota: H_{\text {rad }}^{4}\left(\mathbb{R}^{5}\right) \rightarrow X$ is a continuous embedding.

The second assertion is proved similarly. Indeed, given $f \in X$ we find a sequence $\left(f_{n}\right)_{n \in \mathbb{N}} \subset C_{c}^{\infty}\left(\mathbb{R}^{5}\right)$ such that $f_{n} \rightarrow f$ in $X$. By Lemma 3.12, $\left(f_{n}\right)_{n \in \mathbb{N}}$ is Cauchy in $W^{1, \infty}\left(\mathbb{R}^{5}\right)$ and therefore converges to a limiting function $\hat{f} \in C^{1}\left(\mathbb{R}^{5}\right) \cap W^{1, \infty}\left(\mathbb{R}^{5}\right)$. Using this, we define an inclusion map $\iota: X \rightarrow C^{1}\left(\mathbb{R}^{5}\right) \cap W^{1, \infty}\left(\mathbb{R}^{5}\right)$ by setting $\iota(f):=\hat{f}$. It remains to show that $\iota$ is injective. If $\iota(f)=0$, it follows that there exists a sequence $\left(f_{n}\right)_{n \in \mathbb{N}} \subset C_{c}^{\infty}\left(\mathbb{R}^{5}\right)$ that converges to $f$ in $X$ and to 0 in $L^{\infty}\left(\mathbb{R}^{5}\right)$. Consequently,

$$
\left|\int_{\mathbb{R}^{5}} \Delta f_{n} \varphi\right|=\left|\int_{\mathbb{R}^{5}} f_{n} \Delta \varphi\right| \lesssim\left\|f_{n}\right\|_{L^{\infty}\left(\mathbb{R}^{5}\right)} \rightarrow 0
$$

for any $\varphi \in C_{c}^{\infty}\left(\mathbb{R}^{5}\right)$ and thus, $\Delta f_{n} \rightarrow 0$ in $L^{2}\left(\mathbb{R}^{5}\right)$. Analogously, we obtain $\Delta^{2} f_{n} \rightarrow 0$ in $L^{2}\left(\mathbb{R}^{5}\right)$. By the uniqueness of weak limits we therefore have $\lim _{n \rightarrow \infty}\left\|f_{n}\right\|_{X}=0$ and this shows $f=0$.

Theorem 3.14 The Sobolev space $X$ is invariant under $e^{s \mathcal{L}}$ and there exists a constant $\omega_{0}>0$ such that

$$
\left\|e^{s \mathcal{L}} f\right\|_{X} \lesssim e^{-\omega_{0} s}\|f\|_{X}
$$

for all $s \geq 0$ and all $f \in X$ satisfying $\left(f \mid \psi_{1}\right)_{L_{\sigma}^{2}\left(\mathbb{R}^{5}\right)}=0$. 
Proof By Lemma 3.13, $\mathcal{D}\left(\mathcal{L}^{2}\right) \cap H^{4}\left(\mathbb{R}^{5}\right) \hookrightarrow X$. Since the former space is invariant under $e^{s \mathcal{L}}$, see Lemma 3.8, it follows that $e^{s \mathcal{L}} f \in X$ for all $s \geq 0$ and all $f \in C_{c}^{\infty}\left(\mathbb{R}^{5}\right)$. Consequently, in view of Lemmas 3.9 and 3.10, and a density argument, it suffices to prove the bound

$$
\|f\|_{G\left(\mathcal{L}^{2}\right)} \lesssim\|f\|_{X}
$$

for all $f \in C_{c}^{\infty}\left(\mathbb{R}^{5}\right)$. Thanks to the strong decay of the weight $\sigma(x)=e^{-|x|^{2} / 4}$, we immediately obtain

$$
\begin{aligned}
\|f\|_{G\left(\mathcal{L}^{2}\right)} \lesssim & \left\||\cdot|^{-2} f\right\|_{L^{2}\left(\mathbb{R}^{5}\right)}+\left\||\cdot|^{-1} \nabla f\right\|_{L^{2}\left(\mathbb{R}^{5}\right)}+\|\Delta f\|_{L^{2}\left(\mathbb{R}^{5}\right)} \\
& +\left\||\cdot|^{-1} \nabla \Delta f\right\|_{L^{2}\left(\mathbb{R}^{5}\right)}+\left\|\Delta^{2} f\right\|_{L^{2}\left(\mathbb{R}^{5}\right)} \\
\lesssim & \|f\|_{X}
\end{aligned}
$$

by Hardy's inequality.

\section{The nonlinear evolution}

Now we turn to the full nonlinear problem Eq. (2.5). As before with the linear operator, we switch to 5 -dimensional notation and define the nonlinearity $\mathcal{N}$, acting on functions $f: \mathbb{R}^{5} \rightarrow \mathbb{R}$, by

$\mathcal{N}(f)(x):=-\frac{1}{|x|^{3}}\left[\sin \left(2 f_{0}(|x|)+2|x| f(x)\right)-\sin \left(2 f_{0}(|x|)\right)-2|x| \cos \left(2 f_{0}(|x|)\right) f(x)\right]$.

With this convention, Eq. (2.5) can be written as

$$
\left\{\begin{array}{l}
\partial_{s} \phi(s)=\mathcal{L} \phi(s)+\mathcal{N}(\phi(s)) \\
\phi(0)=\mathcal{U}(h, T)
\end{array}\right.
$$

where

$$
\mathcal{U}(h, T)(x):=f_{0}(\sqrt{T}|x|) /|x|-f_{0}(|x|) /|x|+h(\sqrt{T}|x|) /|x|
$$

and $\phi:[0, \infty) \rightarrow X$.

So far, this is purely formal. In what follows we first prove basic embedding theorems and then some Moser-type inequalities. These will allow us to show that the nonlinearity is locally Lipschitz on $X$. Next, we study mapping properties of the "initial data operator" $\mathcal{U}$ and finally, we implement an infinite-dimensional version of the Lyapunov-Perron method to prove global existence for Eq. (4.1).

\subsection{Further properties of the space $X$}

Corollary 4.1 (Algebra property) We have the bound

$$
\|f g\|_{X} \lesssim\|f\|_{X}\|g\|_{X}
$$

for all $f, g \in X$. As a consequence, $X$ is a Banach algebra.

Proof This is a straightforward consequence of the Leibniz rule, the Gagliardo-Nirenberg inequality (see e.g. [41]), and Lemma 3.12.

Next, we prove weighted $L^{\infty}$ bounds outside of balls. As opposed to Lemma 3.12 and Corollary 4.1, the restriction to radial functions is crucial here. 
Lemma 4.2 We have the bounds

$$
\begin{aligned}
&\left\||\cdot|^{\frac{3}{2}} f\right\|_{L^{\infty}\left(\mathbb{R}^{5} \backslash \mathbb{B}^{5}\right)} \lesssim\|f\|_{\dot{H}^{1}\left(\mathbb{R}^{5}\right)} \\
&\left\||\cdot|^{\frac{1}{2}} f\right\|_{L^{\infty}\left(\mathbb{R}^{5} \backslash \mathbb{B}^{5}\right)} \lesssim\|f\|_{\dot{H}^{2}\left(\mathbb{R}^{5}\right)}
\end{aligned}
$$

for all radial $f \in C_{c}^{\infty}\left(\mathbb{R}^{5}\right)$.

Proof Let $f \in C_{c}^{\infty}\left(\mathbb{R}^{5}\right)$ be radial and write $f(x)=\tilde{f}(|x|)$. The fundamental theorem of calculus yields

$$
\tilde{f}(r)=-\int_{r}^{\infty} \tilde{f}^{\prime}(s) \mathrm{d} s=-\int_{r}^{\infty} s^{-2} \tilde{f}^{\prime}(s) s^{2} \mathrm{~d} s
$$

and thus, by Cauchy-Schwarz,

$$
|\tilde{f}(r)| \leq\left\||\cdot|^{2} \tilde{f}^{\prime}\right\|_{L^{2}(1, \infty)}\left(\int_{r}^{\infty} s^{-4} \mathrm{~d} s\right)^{1 / 2} \lesssim r^{-\frac{3}{2}}\left\||\cdot|^{2} \tilde{f}^{\prime}\right\|_{L^{2}(1, \infty)} \lesssim r^{-\frac{3}{2}}\|\nabla f\|_{L^{2}\left(\mathbb{R}^{5}\right)}
$$

for all $r \geq 1$. This implies the first assertion.

For the second statement we proceed similarly and use

$$
\tilde{f}(r)=\int_{r}^{\infty} \int_{s}^{\infty} \tilde{f}^{\prime \prime}(t) \mathrm{d} t=\int_{r}^{\infty} \int_{s}^{\infty} t^{-2} \tilde{f}^{\prime \prime}(t) t^{2} \mathrm{~d} t
$$

to obtain the bound

$$
|\tilde{f}(r)| \leq\left\||\cdot|^{2} \tilde{f}^{\prime \prime}\right\|_{L^{2}(1, \infty)} \int_{r}^{\infty}\left(\int_{s}^{\infty} t^{-4} \mathrm{~d} t\right)^{1 / 2} \mathrm{~d} s \lesssim r^{-\frac{1}{2}}\left\||\cdot|^{2} \tilde{f}^{\prime \prime}\right\|_{L^{2}(1, \infty)}
$$

for all $r \geq 1$. Now note that

$$
\tilde{f}^{\prime}(|x|)=\frac{x^{j}}{|x|} \partial_{j} f(x)
$$

and thus, by Hardy's inequality, we infer

$$
r^{\frac{1}{2}}|\tilde{f}(r)| \lesssim\|\Delta f\|_{L^{2}\left(\mathbb{R}^{5}\right)}+\left\||\cdot|^{-1} \nabla f\right\|_{L^{2}\left(\mathbb{R}^{5}\right)} \lesssim\|\Delta f\|_{L^{2}\left(\mathbb{R}^{5}\right)}
$$

for all $r \geq 1$, which is the desired result.

\subsection{Nonlinear estimates}

For $\delta>0$ we set

$$
X_{\delta}:=\left\{f \in X:\|f\|_{X} \leq \delta\right\} .
$$

The goal of this section is to prove that the nonlinearity $\mathcal{N}$ is locally Lipschitz on $X$. The key results in this respect are the following Moser-type inequalities. First, we focus on large radii where we need to assume a decay property.

Proposition 4.3 Let $\Phi \in C^{4}\left(\mathbb{R} \times \mathbb{R}^{5}\right)$ and suppose

$$
\left|\partial^{\alpha} \Phi(v, x)\right| \lesssim\langle x\rangle^{-1}
$$

for all $(v, x) \in \mathbb{R} \times \mathbb{R}^{5} \backslash \mathbb{B}^{5}$ and all multi-indices $\alpha \in \mathbb{N}_{0}^{6}$ with $|\alpha| \leq 4$. For $f: \mathbb{R}^{5} \rightarrow \mathbb{R}$ set

$$
\mathcal{M}(f)(x):=f(x)^{2} \Phi(|x| f(x), x) .
$$


Then we have the bound

$$
\|\mathcal{M}(f)-\mathcal{M}(g)\|_{\dot{H}^{2}\left(\mathbb{R}^{5} \backslash \mathbb{B}^{5}\right) \cap \dot{H}^{4}\left(\mathbb{R}^{5} \backslash \mathbb{B}^{5}\right)} \lesssim\left(\|f\|_{X}+\|g\|_{X}\right)\|f-g\|_{X}
$$

for all $f, g \in X_{1} \cap C_{c}^{\infty}\left(\mathbb{R}^{5}\right)$.

Proof Let $f, g \in X_{1} \cap C_{c}^{\infty}\left(\mathbb{R}^{5}\right)$ and set $\mathcal{I}(f)(x):=\Phi(|x| f(x), x)$. Then we have

$$
\mathcal{M}(f)-\mathcal{M}(g)=f^{2} \mathcal{I}(f)-g^{2} \mathcal{I}(g)=\left(f^{2}-g^{2}\right) \mathcal{I}(f)+g^{2}[\mathcal{I}(f)-\mathcal{I}(g)]
$$

and

$$
\begin{aligned}
\mathcal{I}(f)(x)-\mathcal{I}(g)(x) & =\int_{0}^{1} \partial_{t} \Phi(|x| g(x)+t|x|(f(x)-g(x)), x) \mathrm{d} t \\
& =[f(x)-g(x)]|x| \int_{0}^{1} \partial_{1} \Phi(|x| g(x)+t|x|(f(x)-g(x)), x) \mathrm{d} t \\
& =:[f(x)-g(x)] \mathcal{J}(f, g)(x) .
\end{aligned}
$$

Consequently, it suffices to prove

$$
\|g h \mathcal{I}(f)\|_{\dot{H}^{2}(\Omega) \cap \dot{H}^{4}(\Omega)} \lesssim\|g\|_{X}\|h\|_{X}, \quad\left\|g^{2} h \mathcal{J}(f, g)\right\|_{\dot{H}^{2}(\Omega) \cap \dot{H}^{4}(\Omega)} \lesssim\|g\|_{X}^{2}\|h\|_{X}
$$

for all radial $f, g, h \in X_{1} \cap C_{c}^{\infty}\left(\mathbb{R}^{5}\right)$, where $\Omega:=\mathbb{R}^{5} \backslash \mathbb{B}^{5}$.

We start with the estimate for $\mathcal{I}(f)$. By the chain rule,

$$
\begin{aligned}
\langle\cdot\rangle|\nabla \mathcal{I}(f)| & \lesssim|\nabla F|+1 \\
\langle\cdot\rangle|\Delta \mathcal{I}(f)| & \lesssim|\Delta F|+|\nabla F|^{2}+|\nabla F|+1 \\
\langle\cdot\rangle|\nabla \Delta \mathcal{I}(f)| & \lesssim|\nabla \Delta F|+|\Delta F||\nabla F|+|\Delta F|+|\nabla F|^{3}+|\nabla F|^{2}+|\nabla F|+1 \\
\langle\cdot\rangle\left|\Delta^{2} \mathcal{I}(f)\right| & \lesssim\left|\Delta^{2} F\right|+|\nabla \Delta F||\nabla F|+|\nabla \Delta F|+|\Delta F|^{2}+|\Delta F||\nabla F|^{2} \\
& +|\Delta F||\nabla F|+|\Delta F|+|\nabla F|^{4}+|\nabla F|^{3}+|\nabla F|^{2}+|\nabla F|+1,
\end{aligned}
$$

where $F(x)=|x| f(x)$. The strategy is to use Lemma 4.2 to absorb the growing weight in $F$. We consider $\|g h \Delta \mathcal{I}(f)\|_{L^{2}(\Omega)}$ and estimate

$$
\begin{aligned}
\left\|\langle\cdot\rangle^{-1} g h \Delta F\right\|_{L^{2}(\Omega)} & \lesssim\|g h \Delta f\|_{L^{2}(\Omega)}+\left\||\cdot|^{-1} g h \nabla f\right\|_{L^{2}(\Omega)}+\left\||\cdot|^{-2} g h f\right\|_{L^{2}(\Omega)} \\
& \lesssim\|g\|_{X}\|h\|_{X}\|f\|_{X} \\
\left\|\langle\cdot\rangle^{-1} g h|\nabla F|^{2}\right\|_{L^{2}(\Omega)} & \lesssim\left\||\cdot| g h|\nabla f|^{2}\right\|_{L^{2}(\Omega)}+\left\||\cdot|^{-1} g h f^{2}\right\|_{L^{2}(\Omega)} \\
& \lesssim\left\||\cdot|^{\frac{1}{2}} g\right\|_{L^{\infty}(\Omega)}\|h\|_{L^{\infty}(\Omega)}\left\||\cdot|^{\frac{3}{2}} \nabla f\right\|_{L^{\infty}(\Omega)}\left\||\cdot|^{-1} \nabla f\right\|_{L^{2}(\Omega)} \\
& +\left\||\cdot|^{\frac{1}{2}} g\right\|_{L^{\infty}(\Omega)}\left\||\cdot|^{\frac{1}{2}} h\right\|_{L^{\infty}(\Omega)}\|f\|_{L^{\infty}(\Omega)}\left\||\cdot|^{-2} f\right\|_{L^{2}(\Omega)} \\
& \lesssim\|g\| X\|h\|_{X}\|f\|_{X}^{2} \\
\left\|\langle\cdot\rangle^{-1} g h \nabla F\right\|_{L^{2}(\Omega)} & \lesssim\|g h \nabla f\|_{L^{2}(\Omega)}+\left\||\cdot|^{-1} g h f\right\|_{L^{2}(\Omega)} \\
& \lesssim\left\||\cdot|^{\frac{1}{2}} g\right\|_{L^{\infty}(\Omega)}\left\||\cdot|^{\frac{1}{2}} h\right\|_{L^{\infty}(\Omega)}\left(\left\||\cdot|^{-1} \nabla f\right\|_{L^{2}(\Omega)}+\left\||\cdot|^{-2} f\right\|_{L^{2}(\Omega)}\right) \\
& \lesssim\|g\| X\|h\|_{X}\|f\|_{X}
\end{aligned}
$$

by Lemma 4.2 and Hardy's inequality. This yields $\|\Delta[g h \mathcal{I}(f)]\|_{L^{2}(\Omega)} \lesssim\|g\|_{X}\|h\|_{X}$. 
Next, we estimate $\left\|g h \Delta^{2} \mathcal{I}(f)\right\|_{L^{2}(\Omega)}$. The easy terms are

$$
\begin{aligned}
\left\|\langle\cdot\rangle^{-1} \Delta^{2} F\right\|_{L^{2}(\Omega)} & \lesssim \sum_{k=0}^{4}\left\||\cdot|^{-4+k} \nabla^{k} f\right\|_{L^{2}(\Omega)} \lesssim\|f\|_{X} \\
\left\|\langle\cdot\rangle^{-1} \nabla \Delta F \nabla F\right\|_{L^{2}(\Omega)} & \lesssim \sum_{k=0}^{3}\left\||\cdot|^{-2+k} \nabla^{k} f \nabla f\right\|_{L^{2}(\Omega)}+\sum_{k=0}^{3}\left\||\cdot|^{-3+k} \nabla^{k} f f\right\|_{L^{2}(\Omega)} \\
& \lesssim\left(\left\||\cdot|^{\frac{3}{2}} \nabla f\right\|_{L^{\infty}(\Omega)}+\|f\|_{L^{\infty}(\Omega)}\right) \sum_{k=0}^{3}\left\||\cdot|^{-3+k} \nabla^{k} f\right\|_{L^{2}(\Omega)} \\
& \lesssim\|f\|_{X}^{2} \\
\left\|\langle\cdot\rangle^{-1} \nabla \Delta F\right\|_{L^{2}(\Omega)} & \lesssim \sum_{k=0}^{3}\left\||\cdot|^{-3+k} \nabla^{k} f\right\|_{L^{2}(\Omega)} \lesssim\|f\|_{X}
\end{aligned}
$$

as well as

$$
\begin{aligned}
& \left\|\langle\cdot\rangle^{-1} \Delta F|\nabla F|^{2}\right\|_{L^{2}(\Omega)} \\
& \quad \lesssim\left\||\cdot|^{2} \Delta f|\nabla f|^{2}\right\|_{L^{2}(\Omega)}+\left\||\cdot||\nabla f|^{3}\right\|_{L^{2}(\Omega)}+\left\|f|\nabla f|^{2}\right\|_{L^{2}(\Omega)} \\
& \quad+\left\||\cdot| \Delta f f^{2}\right\|_{L^{2}(\Omega)}+\left\|\nabla f f^{2}\right\|_{L^{2}(\Omega)}+\left\||\cdot|^{-1} f^{3}\right\|_{L^{2}(\Omega)} \\
& \quad \lesssim\left\||\cdot|^{\frac{3}{2}} \nabla f\right\|_{L^{\infty}(\Omega)}^{2}\left(\|\Delta f\|_{L^{2}(\Omega)}+\left\||\cdot|^{-1} \nabla f\right\|_{L^{2}(\Omega)}+\left\||\cdot|^{-2} f\right\|_{L^{2}(\Omega)}\right) \\
& \quad+\left\||\cdot|^{\frac{1}{2}} f\right\|_{L^{\infty}(\Omega)}^{2}\left(\|\Delta f\|_{L^{2}(\Omega)}+\left\||\cdot|^{-1} \nabla f\right\|_{L^{2}(\Omega)}+\left\||\cdot|^{-2} f\right\|_{L^{2}(\Omega)}\right) \\
& \quad \lesssim\|f\|_{X}^{3}
\end{aligned}
$$

and

$$
\begin{aligned}
\left\|\langle\cdot\rangle^{-1} \Delta F \nabla F\right\|_{L^{2}(\Omega)} & \lesssim \sum_{k=0}^{2}\left\||\cdot|^{-1+k} \nabla^{k} f \nabla f\right\|_{L^{2}(\Omega)}+\sum_{k=0}^{2}\left\||\cdot|^{-2+k} \nabla^{k} f f\right\|_{L^{2}(\Omega)} \\
& \lesssim\left(\left\||\cdot|^{\frac{3}{2}} \nabla f\right\|_{L^{\infty}(\Omega)}+\|f\|_{L^{\infty}(\Omega)}\right) \sum_{k=0}^{2}\left\||\cdot|^{-2+k} \nabla^{k} f\right\|_{L^{2}(\Omega)} \\
& \lesssim\|f\|_{X}^{2} \\
\left\|\langle\cdot\rangle^{-1}|\nabla F|^{4}\right\|_{L^{2}(\Omega)} & \lesssim\left\||\cdot|^{3}|\nabla f|^{4}\right\|_{L^{2}(\Omega)}+\left\||\cdot|^{-1} f^{4}\right\|_{L^{2}(\Omega)} \\
& \lesssim\left\||\cdot|^{\frac{3}{2}} \nabla f\right\|_{L^{\infty}(\Omega)}^{3}\left\||\cdot|^{-1} \nabla f\right\|_{L^{2}(\Omega)}+\left\||\cdot|^{\frac{1}{2}} f\right\|_{L^{\infty}(\Omega)}^{3}\left\||\cdot|^{-2} f\right\|_{L^{2}(\Omega)} \\
& \lesssim\|f\|_{X}^{4} .
\end{aligned}
$$

Analogously, we estimate

$$
\begin{aligned}
\left\|\langle\cdot\rangle^{-1}|\nabla F|^{3}\right\|_{L^{2}(\Omega)} & \lesssim\left\||\cdot|^{2}|\nabla f|^{3}\right\|_{L^{2}(\Omega)}+\left\||\cdot|^{-1} f^{3}\right\|_{L^{2}(\Omega)} \\
& \lesssim\left\||\cdot|^{\frac{3}{2}} \nabla f\right\|_{L^{\infty}(\Omega)}^{2}\left\||\cdot|^{-1} \nabla f\right\|_{L^{2}(\Omega)}+\left\||\cdot|^{\frac{1}{2}} f\right\|_{L^{\infty}(\Omega)}^{2}\left\||\cdot|^{-2} f\right\|_{L^{2}(\Omega)} \\
& \lesssim\|f\|_{X}^{2} .
\end{aligned}
$$

It remains to control the most delicate term, $|\Delta F|^{2}$. For this one we use Hardy and Lemma 4.2 to obtain 


$$
\begin{aligned}
\left\|\langle\cdot\rangle^{-1} g h|\Delta F|^{2}\right\|_{L^{2}(\Omega)} \lesssim & \left\||\cdot| g h|\Delta f|^{2}\right\|_{L^{2}(\Omega)}+\left\||\cdot|^{-1} g h|\nabla f|^{2}\right\|_{L^{2}(\Omega)}+\left\||\cdot|^{-3} g h f^{2}\right\|_{L^{2}(\Omega)} \\
\lesssim & \left\||\cdot|^{\frac{1}{2}} g\right\|_{L^{\infty}(\Omega)}\left\||\cdot|^{\frac{1}{2}} h\right\|_{L^{\infty}(\Omega)} \\
& \times\left(\left\||\Delta f|^{2}\right\|_{L^{2}(\Omega)}+\left\||\cdot|^{-1}|\nabla f|^{2}\right\|_{L^{2}(\Omega)}+\left\||\cdot|^{-2} f^{2}\right\|_{L^{2}(\Omega)}\right) \\
& \lesssim\|g\|_{X}\|h\|_{X}\|f\|_{X}^{2} .
\end{aligned}
$$

The above estimates easily imply $\left\|\Delta^{2}[g h \mathcal{I}(f)]\right\|_{L^{2}(\Omega)} \lesssim\|g\|_{X}\|h\|_{X}$. Putting everything together, we arrive at the desired $\|g h \mathcal{I}(f)\|_{\dot{H}^{2}(\Omega) \cap \dot{H}^{4}(\Omega)} \lesssim\|g\|_{X}\|h\|_{X}$. The bound on $\mathcal{J}(f, g)$ is proved in the exact same way.

The next bound controls the nonlinearity near the center. Here the issue is to handle powers of $|\cdot|^{-1}$ that arise by differentiation.

Lemma 4.4 Let $\Phi \in C^{4}(\mathbb{R})$ and suppose $\Phi^{\prime}(0)=0$. For $f: \mathbb{R}^{5} \rightarrow \mathbb{R}$ set

$$
\mathcal{M}(f)(x)=f(x)^{2} \Phi(|x| f(x))
$$

Then we have

$$
\|\mathcal{M}(f)-\mathcal{M}(g)\|_{\dot{H}^{2}\left(\mathbb{B}^{5}\right) \cap \dot{H}^{4}\left(\mathbb{B}^{5}\right)} \lesssim\left(\|f\|_{X}+\|g\|_{X}\right)\|f-g\|_{X}
$$

for all $f, g \in X_{1} \cap C_{c}^{\infty}\left(\mathbb{R}^{5}\right)$.

Proof As in the proof of Proposition 4.3, we write $\mathcal{I}(f)(x)=\Phi(|x| f(x))$ and $\mathcal{I}(f)-\mathcal{I}(g)=$ $(f-g) \mathcal{J}(f, g)$ with

$$
\mathcal{J}(f, g)(x)=|x| \int_{0}^{1} \Phi^{\prime}(|x| g(x)+t|x|(f(x)-g(x))) \mathrm{d} t
$$

and it suffices to show

$$
\|\mathcal{I}(f)\|_{\dot{H}^{2}\left(\mathbb{B}^{5}\right) \cap \dot{H}^{4}\left(\mathbb{B}^{5}\right)}+\|\mathcal{J}(f, g)\|_{\dot{H}^{2}\left(\mathbb{B}^{5}\right) \cap \dot{H}^{4}\left(\mathbb{B}^{5}\right)} \lesssim 1
$$

for all $f, g \in X_{1} \cap C_{c}^{\infty}\left(\mathbb{R}^{5}\right)$. We begin with the bound on $\mathcal{I}(f)$. By the chain rule we infer

$$
\begin{aligned}
|\mathcal{I}(f)| & \lesssim 1 \\
|\nabla \mathcal{I}(f)| & \lesssim\left|\Phi^{\prime} \circ F\right||\nabla F| \\
|\Delta \mathcal{I}(f)| & \lesssim\left|\Phi^{\prime} \circ F\right||\Delta F|+|\nabla F|^{2} \\
|\nabla \Delta \mathcal{I}(f)| & \lesssim\left|\Phi^{\prime} \circ F\right||\nabla \Delta F|+|\Delta F||\nabla F|+|\nabla F|^{3} \\
\left|\Delta^{2} \mathcal{I}(f)\right| & \lesssim\left|\Phi^{\prime} \circ F\right|\left|\Delta^{2} F\right|+|\nabla \Delta F||\nabla F|+|\Delta F|^{2}+|\Delta F||\nabla F|^{2}+|\nabla F|^{4}
\end{aligned}
$$

on the ball $\mathbb{B}^{5}$, where $F(x):=|x| f(x)$ and we have used the fact that $\|F\|_{L^{\infty}\left(\mathbb{B}^{5}\right)} \lesssim 1$ which follows from Lemma 3.12. We consider $\|\Delta \mathcal{I}(f)\|_{L^{2}\left(\mathbb{B}^{5}\right)}$ and estimate

$$
\begin{aligned}
\left\|\left(\Phi^{\prime} \circ F\right) \Delta F\right\|_{L^{2}\left(\mathbb{B}^{5}\right)} & \lesssim\|\Delta f\|_{L^{2}\left(\mathbb{B}^{5}\right)}+\|\nabla f\|_{L^{2}\left(\mathbb{B}^{5}\right)}+\left\||\cdot|^{-1} f\right\|_{L^{2}\left(\mathbb{B}^{5}\right)} \\
& \lesssim\|\Delta f\|_{L^{2}\left(\mathbb{R}^{5}\right)}+\left\||\cdot|^{-1} \nabla f\right\|_{L^{2}\left(\mathbb{R}^{5}\right)}+\left\||\cdot|^{-2} f\right\|_{L^{2}\left(\mathbb{R}^{5}\right)} \\
& \lesssim\|f\|_{X} \\
\left\||\nabla F|^{2}\right\|_{L^{2}\left(\mathbb{B}^{5}\right)} & \lesssim\left\||\nabla f|^{2}\right\|_{L^{2}\left(\mathbb{B}^{5}\right)}+\left\|f^{2}\right\|_{L^{2}\left(\mathbb{B}^{5}\right)} \\
& \lesssim\|\nabla f\|_{L^{\infty}\left(\mathbb{R}^{5}\right)}\left\||\cdot|^{-1} \nabla f\right\|_{L^{2}\left(\mathbb{R}^{5}\right)}+\|f\|_{L^{\infty}\left(\mathbb{R}^{5}\right)}\left\||\cdot|^{-2} f\right\|_{L^{2}\left(\mathbb{R}^{5}\right)} \\
& \lesssim\|f\|_{X}^{2}
\end{aligned}
$$


by Lemma 3.12 and Hardy's inequality. This yields the desired $\|\Delta \mathcal{I}(f)\|_{L^{2}\left(\mathbb{B}^{5}\right)} \lesssim 1$.

Next, we estimate $\left\|\Delta^{2} \mathcal{I}(f)\right\|_{L^{2}\left(\mathbb{B}^{5}\right)}$. The most delicate term is $\left|\Phi^{\prime} \circ F \| \Delta^{2} F\right|$ where we absorb one singular factor $|\cdot|^{-1}$ by exploiting the assumption $\Phi^{\prime}(0)=0$. More precisely,

$$
\begin{aligned}
\left\|\left(\Phi^{\prime} \circ F\right) \Delta^{2} F\right\|_{L^{2}\left(\mathbb{B}^{5}\right)} & \left.\left.\lesssim\|\| \cdot\right|^{-1} \Phi^{\prime} \circ F\left\|_{L^{\infty}\left(\mathbb{B}^{5}\right)} \sum_{k=0}^{4}\right\| \cdot\right|^{-2+k} \nabla^{k} f \|_{L^{2}\left(\mathbb{B}^{5}\right)} \\
& \lesssim\|f\|_{X}^{2}
\end{aligned}
$$

by Hardy's inequality, the bound

$$
\left|\left(\Phi^{\prime} \circ F\right)(x)\right|=\left|\Phi^{\prime}(|x| f(x))\right| \lesssim|x||f(x)|,
$$

which follows from $\Phi^{\prime}(0)=0$, and Lemma 3.12. Furthermore, by Gagliardo-Nirenberg (see e.g. [41], p. 8, Proposition 3.1),

$$
\begin{aligned}
\left\||\Delta F|^{2}\right\|_{L^{2}\left(\mathbb{B}^{5}\right)} & \lesssim\left\|(\chi \Delta F)^{2}\right\|_{L^{2}\left(\mathbb{R}^{5}\right)} \lesssim\|\nabla(\chi \nabla F) \nabla(\chi \nabla F)\|_{L^{2}\left(\mathbb{B}^{5}\right)}+\left\|(\nabla \chi \nabla F)^{2}\right\|_{L^{2}\left(\mathbb{R}^{5}\right)} \\
& \lesssim\|\nabla(\chi \nabla F)\|_{L^{4}\left(\mathbb{R}^{5}\right)}^{2}+\left\|(\nabla \chi \nabla F)^{2}\right\|_{L^{2}\left(\mathbb{R}^{5}\right)} \\
& \lesssim\|\chi \nabla F\|_{L^{\infty}\left(\mathbb{R}^{5}\right)}\|\Delta(\chi \nabla F)\|_{L^{2}\left(\mathbb{R}^{5}\right)}+\|\nabla \chi \nabla F\|_{L^{\infty}\left(\mathbb{R}^{5}\right)}^{2} \\
& \lesssim\|f\|_{X}^{2},
\end{aligned}
$$

where $\chi: \mathbb{R}^{5} \rightarrow[0,1]$ is a smooth cut-off satisfying $\chi(x)=1$ for $|x| \leq 1$ and $\chi(x)=0$ for $|x| \geq 2$. The remaining terms are readily estimated as

$$
\begin{aligned}
\|\nabla \Delta F \nabla F\|_{L^{2}\left(\mathbb{B}^{5}\right)} & \lesssim\|\nabla F\|_{L^{\infty}\left(\mathbb{B}^{5}\right)}\|\nabla \Delta F\|_{L^{2}\left(\mathbb{B}^{5}\right)} \lesssim\|f\|_{X}^{2} \\
\left\|\Delta F|\nabla F|^{2}\right\|_{L^{2}\left(\mathbb{B}^{5}\right)} & \lesssim\|\nabla F\|_{L^{\infty}\left(\mathbb{B}^{5}\right)}^{2}\|\Delta F\|_{L^{2}\left(\mathbb{B}^{5}\right)} \lesssim\|f\|_{X}^{3}
\end{aligned}
$$

This shows $\left\|\Delta^{2} \mathcal{I}(f)\right\|_{L^{2}\left(\mathbb{B}^{5}\right)} \lesssim 1$. The proof of the bound on $\mathcal{J}(f, g)$ is identical.

In fact, we need a slightly more general form of Lemma 4.4.

Corollary 4.5 Let $\Phi \in C^{4}\left(\mathbb{R}^{2}\right)$ and suppose $\partial_{1} \Phi(0,0)=\partial_{2} \Phi(0,0)=0$. Set

$$
\mathcal{M}(f)(x)=f(x)^{2} \Phi\left(|x| f(x),|x| \varphi_{0}(x)\right)
$$

where $\varphi_{0} \in C_{c}^{\infty}\left(\mathbb{R}^{5}\right)$ is a fixed function. Then we have

$$
\|\mathcal{M}(f)-\mathcal{M}(g)\|_{\dot{H}^{2}\left(\mathbb{B}^{5}\right) \cap \dot{H}^{4}\left(\mathbb{B}^{5}\right)} \lesssim\left(\|f\|_{X}+\|g\|_{X}\right)\|f-g\|_{X}
$$

for all $f \in X_{1} \cap C_{c}^{\infty}\left(\mathbb{R}^{5}\right)$.

Proof This is a straightforward generalization of Lemma 4.4.

We are now in a position to prove that the nonlinearity $\mathcal{N}$ is locally Lipschitz on $X$.

Lemma 4.6 We have the bound

$$
\|\mathcal{N}(f)-\mathcal{N}(g)\| \lesssim\left(\|f\|_{X}+\|g\|_{X}\right)\|f-g\|_{X}
$$

for all $f, g \in X_{1}$. 
Proof By a density argument it suffices to consider $f, g \in X_{1} \cap C_{c}^{\infty}\left(\mathbb{R}^{5}\right)$. Recall that $\mathcal{N}(f)(x)=N(f(x),|x|)$ with

$$
N(u, y)=-\frac{1}{y^{3}}\left[\sin \left(2 f_{0}(y)+2 y u\right)-\sin \left(2 f_{0}(y)\right)-2 y \cos \left(2 f_{0}(y)\right) u\right] .
$$

Note that

$$
\begin{aligned}
& \partial_{1} N(u, y)=-\frac{2}{y^{2}}\left[\cos \left(2 f_{0}(y)+2 y u\right)-\cos \left(2 f_{0}(y)\right)\right] \\
& \partial_{1}^{2} N(u, y)=\frac{4}{y} \sin \left(2 f_{0}(y)+2 y u\right) \\
& \partial_{1}^{3} N(u, y)=8 \cos \left(2 f_{0}(y)+2 y u\right) .
\end{aligned}
$$

Evidently, $N(0, y)=\partial_{1} N(0, y)=0$ and the fundamental theorem of calculus yields

$$
\begin{aligned}
N(u, y)= & \int_{0}^{1} \partial_{t_{1}} N\left(t_{1} u, y\right) \mathrm{d} t_{1}=u \int_{0}^{1} \partial_{1} N\left(t_{1} u, y\right) \mathrm{d} t_{1}=u^{2} \int_{0}^{1} t_{1} \int_{0}^{1} \partial_{1}^{2} N\left(t_{2} t_{1} u, y\right) \mathrm{d} t_{2} \mathrm{~d} t_{1} \\
= & \frac{4 u^{2}}{y} \int_{0}^{1} t_{1} \int_{0}^{1} \sin \left(2 f_{0}(y)+2 t_{2} t_{1} y u\right) \mathrm{d} t_{2} \mathrm{~d} t_{1} \\
= & 8 u^{2} \frac{f_{0}(y)}{y} \int_{0}^{1} t_{1} \int_{0}^{1} \int_{0}^{1} \cos \left(2 t_{3} f_{0}(y)+2 t_{3} t_{2} t_{1} y u\right) \mathrm{d} t_{3} \mathrm{~d} t_{2} \mathrm{~d} t_{1} \\
& +8 u^{3} \int_{0}^{1} t_{1}^{2} \int_{0}^{1} t_{2} \int_{0}^{1} \cos \left(2 t_{3} f_{0}(y)+2 t_{3} t_{2} t_{1} y u\right) \mathrm{d} t_{3} \mathrm{~d} t_{2} \mathrm{~d} t_{1} .
\end{aligned}
$$

We define $\Phi_{1}, \tilde{\Phi}_{1}: \mathbb{R}^{2} \rightarrow \mathbb{R}$ and $\Phi_{2}: \mathbb{R} \times \mathbb{R}^{5} \rightarrow \mathbb{R}$ by

$$
\begin{aligned}
\Phi_{1}\left(v, v_{0}\right) & :=8 \int_{0}^{1} t_{1} \int_{0}^{1} \int_{0}^{1} \cos \left(2 t_{3} v_{0}+2 t_{3} t_{2} t_{1} v\right) \mathrm{d} t_{3} \mathrm{~d} t_{2} \mathrm{~d} t_{1} \\
\tilde{\Phi}_{1}\left(v, v_{0}\right) & :=8 \int_{0}^{1} t_{1}^{2} \int_{0}^{1} t_{2} \int_{0}^{1} \cos \left(2 t_{3} v_{0}+2 t_{3} t_{2} t_{1} v\right) \mathrm{d} t_{3} \mathrm{~d} t_{2} \mathrm{~d} t_{1} \\
\Phi_{2}(v, x) & :=\frac{4}{|x|} \int_{0}^{1} t_{1} \int_{0}^{1} \sin \left(2 f_{0}(|x|)+2 t_{2} t_{1} v\right) \mathrm{d} t_{2} \mathrm{~d} t_{1}
\end{aligned}
$$

and set

$$
\begin{aligned}
& \mathcal{M}_{1}(f)(x):=f(x)^{2} \Phi_{1}\left(|x| f(x),|x| \varphi_{0}(x)\right) \\
& \tilde{\mathcal{M}}_{1}(f)(x):=f(x)^{2} \tilde{\Phi}_{1}\left(|x| f(x),|x| \varphi_{0}(x)\right) \\
& \mathcal{M}_{2}(f)(x):=f(x)^{2} \Phi_{2}(|x| f(x), x)
\end{aligned}
$$

where $\varphi_{0}(x):=\chi(x) f_{0}(|x|) /|x|$ with $\chi: \mathbb{R}^{5} \rightarrow[0,1]$ the usual smooth cut-off satisfying $\chi(x)=1$ for $|x| \leq 1$ and $\chi(x)=0$ for $|x| \geq 2$. By [3], $f_{0}$ is odd and thus, $\varphi_{0} \in C_{c}^{\infty}\left(\mathbb{R}^{5}\right)$. This yields the representations

$$
\mathcal{N}(f)(x)=\varphi_{0}(x) \mathcal{M}_{1} f(x)+f(x) \tilde{\mathcal{M}}_{1}(f)(x)
$$

for $|x| \leq 1$ and

$$
\mathcal{N}(f)(x)=\mathcal{M}_{2}(f)(x)
$$

for $|x| \geq \frac{1}{2}$. Evidently, we have

$$
\partial_{1} \Phi_{1}(0,0)=\partial_{2} \Phi_{1}(0,0)=\partial_{1} \tilde{\Phi}_{1}(0,0)=\partial_{2} \tilde{\Phi}_{1}(0,0)=0
$$


and

$$
\left|\partial^{\alpha} \Phi_{2}(v, x)\right| \leq C_{\alpha}\langle x\rangle^{-1}
$$

for all $(v, x) \in \mathbb{R} \times \mathbb{R}^{5} \backslash \mathbb{B}^{5}$ and all multi-indices $\alpha \in \mathbb{N}_{0}^{6}$. As a consequence, Corollary 4.5 and Proposition 4.3 apply to $\mathcal{M}_{1}, \tilde{\mathcal{M}}_{1}$, and $\mathcal{M}_{2}$, respectively, and Corollary 4.1 yields the claim.

\subsection{The initial data operator}

Now we consider the initial data operator

$$
\mathcal{U}(h, T)(x):=f_{0}(\sqrt{T}|x|) /|x|-f_{0}(|x|) /|x|+h(\sqrt{T}|x|) /|x| .
$$

Recall that

$$
\tilde{Y}=\left\{h \in C_{c}^{\infty}([0, \infty)): h^{(2 k)}(0)=0 \text { for all } k \in \mathbb{N}_{0}\right\}
$$

and $Y$ was defined as the completion of $\tilde{Y}$ with respect to the norm

$$
\|h\|_{Y}=\left\||\cdot|^{-1} h(|\cdot|)\right\|_{X} .
$$

First, we need to make sure that $\mathcal{U}(h, T)$ has values in $X$. The following more general result will be helpful in this respect.

Lemma 4.7 Let $f \in C^{\infty}\left(\mathbb{R}^{5}\right)$ and assume the bounds

$$
\left|\nabla^{k} f(x)\right| \lesssim\langle x\rangle^{-1-k}
$$

for all $x \in \mathbb{R}^{5}$ and $k \in\{0,1,2,3,4\}$. Then $f \in X$.

Proof Let $\chi: \mathbb{R}^{5} \rightarrow[0,1]$ be the usual smooth cut-off satisfying $\chi(x)=1$ for $|x| \leq 1$ and $\chi(x)=0$ for $|x| \geq 2$. For $n \in \mathbb{N}$ we set $\chi_{n}(x):=\chi(x / n)$. Then $\chi_{n} f \in C_{c}^{\infty}\left(\mathbb{R}^{5}\right)$ for any $n \in \mathbb{N}$ and $\chi_{n} f-f=0$ on $\mathbb{B}_{n}^{5}$. Thus, thanks to the decay $|f(x)| \lesssim\langle x\rangle^{-1}$,

$$
\begin{aligned}
\left\|\chi_{n} f-f\right\|_{L^{\infty}\left(\mathbb{R}^{5}\right)} & \leq\left\|\chi_{n} f-f\right\|_{L^{\infty}\left(\mathbb{B}_{n}^{5}\right)}+\left\|\chi_{n} f-f\right\|_{L^{\infty}\left(\mathbb{R}^{5} \backslash \mathbb{B}_{n}^{5}\right)} \lesssim\|f\|_{L^{\infty}\left(\mathbb{R}^{5} \backslash \mathbb{B}_{n}^{5}\right)} \\
& \lesssim n^{-1}
\end{aligned}
$$

and we see that $\chi_{n} f \rightarrow f$ in $L^{\infty}\left(\mathbb{R}^{5}\right)$. Now let $m \leq n$ and note that $\chi_{n} f-\chi_{m} f=0$ on the ball $\mathbb{B}_{m}^{5}$. Furthermore,

$$
\begin{aligned}
\left\|\chi_{n} f\right\|_{\dot{H}^{2}\left(\mathbb{R}^{5} \backslash \mathbb{B}_{m}^{5}\right)} & \simeq\left\|\Delta \chi_{n} f\right\|_{L^{2}\left(\mathbb{R}^{5} \backslash \mathbb{B}_{m}^{5}\right)}+\left\|\nabla \chi_{n} \nabla f\right\|_{L^{2}\left(\mathbb{R}^{5} \backslash \mathbb{B}_{m}^{5}\right)}+\left\|\chi_{n} \Delta f\right\|_{L^{2}\left(\mathbb{R}^{5} \backslash \mathbb{B}_{m}^{5}\right)} \\
& \lesssim\left\||\cdot|^{-1} \Delta \chi_{n}\right\|_{L^{2}\left(\mathbb{R}^{5} \backslash \mathbb{B}_{m}^{5}\right)}+\left\||\cdot|^{-2} \nabla \chi_{n}\right\|_{L^{2}\left(\mathbb{R}^{5} \backslash \mathbb{B}_{m}^{5}\right)}+\left\||\cdot|^{-3} \chi_{n}\right\|_{L^{2}\left(\mathbb{R}^{5} \backslash \mathbb{B}_{m}^{5}\right)} \\
& \lesssim m^{-\frac{1}{2}}
\end{aligned}
$$

and similarly for $\left\|\chi_{n} f\right\|_{\dot{H}^{4}\left(\mathbb{R}^{5} \backslash \mathbb{B}_{m}^{5}\right)}$. In summary, we find

$$
\left\|\chi_{m} f-\chi_{n} f\right\|_{X} \lesssim m^{-\frac{1}{2}}+n^{-\frac{1}{2}}
$$

for all $n, m \in \mathbb{N}$ and thus, $\left(\chi_{n} f\right)_{n \in \mathbb{N}}$ is Cauchy in $X$. This shows $f \in X$, cf. Lemma 3.13.

Corollary 4.8 The function $x \mapsto f_{0}(|x|) /|x|: \mathbb{R}^{5} \rightarrow \mathbb{R}$ belongs to $X$. 
Proof By [3], $f_{0}:[0, \infty) \rightarrow \mathbb{R}$ is smooth, odd, and satisfies the bounds $\left|f_{0}^{(k)}(y)\right| \lesssim y^{-k}$ for all $y \geq 1$ and $k \in\{0,1,2,3,4\}$. Consequently, the function $x \mapsto f_{0}(|x|) /|x|: \mathbb{R}^{5} \rightarrow \mathbb{R}$ verifies the hypotheses of Lemma 4.7.

Lemma 4.9 The map $\mathcal{U}: Y \times\left[\frac{1}{2}, \frac{3}{2}\right] \rightarrow X$ is well-defined and continuous. Furthermore, we have the bound

$$
\|\mathcal{U}(h, T)\|_{X} \lesssim\|h\|_{Y}+|T-1|
$$

for all $(h, T) \in Y \times\left[\frac{1}{2}, \frac{3}{2}\right]$.

Proof Corollary 4.8 and the very definition of $Y$ show that $\mathcal{U}$ has values in $X$. Hence, $\mathcal{U}$ is well-defined. For brevity we set $I:=\left[\frac{1}{2}, \frac{3}{2}\right], \varphi_{0}(x):=f_{0}(|x|) /|x|$, and $H(x):=h(|x|) /|x|$. Since $\|h\|_{Y}=\|H\|_{X}$, we have to show that the maps $T \mapsto \varphi_{0}(\sqrt{T}(\cdot)): I \rightarrow X$ and $(H, T) \mapsto H(\sqrt{T}(\cdot)): X \times I \rightarrow X$ are continuous. The fundamental theorem of calculus yields

$$
\begin{aligned}
\varphi_{0}\left(\sqrt{T_{1}} x\right)-\varphi_{0}\left(\sqrt{T_{2}} x\right) & =\int_{0}^{1} \partial_{t} \varphi_{0}\left(\sqrt{T_{2}} x+t\left(\sqrt{T_{1}}-\sqrt{T_{2}}\right) x\right) \mathrm{d} t \\
& =\left(\sqrt{T_{1}}-\sqrt{T_{2}}\right) \int_{0}^{1} x \nabla \varphi_{0}\left(\sqrt{T_{2}} x+t\left(\sqrt{T_{1}}-\sqrt{T_{2}}\right) x\right) \mathrm{d} t \\
& =:\left(\sqrt{T_{1}}-\sqrt{T_{2}}\right) \psi_{T_{1}, T_{2}}(x) .
\end{aligned}
$$

Obviously, $\psi_{T_{1}, T_{2}} \in C^{\infty}\left(\mathbb{R}^{5}\right)$ and $\left|\nabla^{k} \psi_{T_{1}, T_{2}}(x)\right| \lesssim\langle x\rangle^{-1-k}$ for all $x \in \mathbb{R}^{5}$ and $k \in$ $\{0,1,2,3,4\}$. Consequently, $\psi_{T_{1}, T_{2}} \in X$ by Lemma 4.7 and we infer

$$
\left\|\varphi_{0}\left(\sqrt{T_{1}}(\cdot)\right)-\varphi_{0}\left(\sqrt{T_{2}}(\cdot)\right)\right\|_{X} \leq\left|\sqrt{T_{1}}-\sqrt{T_{2}}\right|\left\|\psi_{T_{1}, T_{2}}\right\|_{X} \lesssim\left|\sqrt{T_{1}}-\sqrt{T_{2}}\right|
$$

for all $T_{1}, T_{2} \in I$.

Now let $\epsilon>0$ and choose $H_{1}, H_{2} \in X$ such that $\left\|H_{1}-H_{2}\right\|_{X} \leq \epsilon / 100$. Furthermore, choose $\tilde{H}_{1} \in C_{c}^{\infty}\left(\mathbb{R}^{5}\right)$ such that $\left\|H_{1}-\tilde{H}_{1}\right\|_{X} \leq \epsilon / 100$. Then we have

$$
\begin{aligned}
\left\|H_{1}\left(\sqrt{T_{1}}(\cdot)\right)-H_{2}\left(\sqrt{T_{2}}(\cdot)\right)\right\|_{X} \leq & \left\|H_{1}\left(\sqrt{T_{1}}(\cdot)\right)-H_{1}\left(\sqrt{T_{2}}(\cdot)\right)\right\|_{X} \\
& +\left\|H_{1}\left(\sqrt{T_{2}}(\cdot)\right)-H_{2}\left(\sqrt{T_{2}}(\cdot)\right)\right\|_{X} \\
\leq & \left\|H_{1}\left(\sqrt{T_{1}}(\cdot)\right)-H_{1}\left(\sqrt{T_{2}}(\cdot)\right)\right\|_{X}+\frac{\epsilon}{4}
\end{aligned}
$$

and

$$
\begin{aligned}
\left\|H_{1}\left(\sqrt{T_{1}}(\cdot)\right)-H_{1}\left(\sqrt{T_{2}}(\cdot)\right)\right\|_{X} \leq & \left\|H_{1}\left(\sqrt{T_{1}}(\cdot)\right)-\tilde{H}_{1}\left(\sqrt{T_{1}}(\cdot)\right)\right\|_{X} \\
& +\left\|\tilde{H}_{1}\left(\sqrt{T_{1}}(\cdot)\right)-\tilde{H}_{1}\left(\sqrt{T_{2}}(\cdot)\right)\right\|_{X} \\
& +\left\|\tilde{H}_{1}\left(\sqrt{T_{2}}(\cdot)\right)-H_{1}\left(\sqrt{T_{2}}(\cdot)\right)\right\|_{X} \\
\leq & \frac{\epsilon}{2}+C_{\epsilon}\left|\sqrt{T_{1}}-\sqrt{T_{2}}\right|
\end{aligned}
$$

for all $T_{1}, T_{2} \in I$ again by the fundamental theorem of calculus. Consequently, we may choose $\left|T_{1}-T_{2}\right|$ so small that

$$
\left\|H_{1}\left(\sqrt{T_{1}}(\cdot)\right)-H_{2}\left(\sqrt{T_{2}}(\cdot)\right)\right\|_{X}<\epsilon .
$$


This proves the continuity of $\mathcal{U}$. Finally, from the above it is obvious that

$$
\|\mathcal{U}(h, T)\|_{X} \lesssim\|h\|_{Y}+|\sqrt{T}-1| \lesssim\|h\|_{Y}+|T-1| .
$$

\subsection{Global existence for the modified equation}

Now we turn to the solution of Eq. (4.1). As an intermediate step we consider the Cauchy problem

$$
\left\{\begin{array}{l}
\partial_{s} \phi(s)=\mathcal{L} \phi(s)+\mathcal{N}(\phi(s)) \\
\phi(0)=f
\end{array}\right.
$$

for given small $f \in X$. We employ Duhamel's principle to obtain the weak formulation

$$
\phi(s)=e^{s \mathcal{L}} f+\int_{0}^{s} e^{\left(s-s^{\prime}\right) \mathcal{L}} \mathcal{N}\left(\phi\left(s^{\prime}\right)\right) \mathrm{d} s^{\prime} .
$$

As a matter of fact, this equation does not have global solutions for arbitrary $f$ due to the unstable subspace of the semigroup $e^{s \mathcal{L}}$. Thus, we modify Eq. (4.3) by adding a correction term that stabilizes the evolution. In order to obtain this term, we formally project the evolution to the unstable subspace. That is to say, we define the projection operator $\mathcal{P}: H \rightarrow H$ by

$$
\mathcal{P} f:=\left(f \mid \psi_{1}\right)_{L_{\sigma}^{2}\left(\mathbb{R}^{5}\right)} \psi_{1} .
$$

Note that by [3], $\psi_{1}(x)=f_{0}^{\prime}(|x|) /\left\|f_{0}^{\prime}(|\cdot|)\right\|_{L_{\sigma}^{2}\left(\mathbb{R}^{5}\right)}$ satisfies the assumptions of Lemma 4.7 and thus, $\mathcal{P}$ has values in $X$. Furthermore, by Lemma 3.12, $\left.\mathcal{P}\right|_{X}$ is a bounded projection on $X$. Applying $\mathcal{P}$ to Eq. (4.3) and using the fact that $\mathcal{P} e^{s \mathcal{L}} f=e^{s} \mathcal{P} f$, we obtain (at least formally)

$$
\mathcal{P} \phi(s)=e^{s} \mathcal{P} f+e^{s} \int_{0}^{s} e^{-s^{\prime}} \mathcal{P N}\left(\phi\left(s^{\prime}\right)\right) \mathrm{d} s^{\prime} .
$$

This suggests to subtract the term $e^{s} \mathcal{C}(\Phi, f)$, where

$$
\mathcal{C}(\phi, f):=\mathcal{P} f+\int_{0}^{\infty} e^{-s^{\prime}} \mathcal{P} \mathcal{N}\left(\phi\left(s^{\prime}\right)\right) \mathrm{d} s^{\prime} .
$$

In order to put this on a sound functional analytic footing, we introduce the Banach space

$$
\mathcal{X}:=\{\phi \in C([0, \infty), X):\|\phi\| \mathcal{X}<\infty\}
$$

with the norm

$$
\|\phi\|_{\mathcal{X}}:=\sup _{s>0} e^{\omega_{0} s}\|\phi(s)\|_{X}
$$

where $\omega_{0}>0$ is the constant from Theorem 3.14. Furthermore, for $\delta>0$, we set

$$
\mathcal{X}_{\delta}:=\{\phi \in \mathcal{X}:\|\phi\| \mathcal{X} \leq \delta\} .
$$

Now we define $\mathcal{K}: \mathcal{X} \times X \rightarrow \mathcal{X}$ by

$$
\mathcal{K}(\phi, f)(s):=e^{s \mathcal{L}} f+\int_{0}^{s} e^{\left(s-s^{\prime}\right) \mathcal{L}} \mathcal{N}\left(\phi\left(s^{\prime}\right)\right) \mathrm{d} s^{\prime}-e^{s} \mathcal{C}(\phi, f)
$$

and show that $\mathcal{K}(\cdot, f)$ is a contraction on $\mathcal{X}_{\delta}$, provided $f \in X$ is sufficiently small. 
Lemma 4.10 There exists a constant $c>0$ such that $\mathcal{K}(\phi, f) \in \mathcal{X}_{\delta}$ for all $\phi \in \mathcal{X}_{\delta}$ and all $f \in X_{\delta / c}$, provided $\delta>0$ is sufficiently small.

Proof By definition, we have

$$
\mathcal{P K}(\phi, f)(s)=-e^{s} \int_{s}^{\infty} e^{-s^{\prime}} \mathcal{P} \mathcal{N}\left(\phi\left(s^{\prime}\right)\right) \mathrm{d} s^{\prime}
$$

and thus, by Lemma 4.6,

$$
\|\mathcal{P K}(\phi, f)(s)\|_{X} \lesssim e^{s} \int_{s}^{\infty} e^{-s^{\prime}}\left\|\phi\left(s^{\prime}\right)\right\|_{X}^{2} \mathrm{~d} s^{\prime} \lesssim e^{-2 \omega_{0} s}\|\phi\|_{\mathcal{X}}^{2} \lesssim \delta^{2} e^{-2 \omega_{0} s}
$$

Similarly,

$$
(1-\mathcal{P}) \mathcal{K}(\phi, f)(s)=e^{s \mathcal{L}}(1-\mathcal{P}) f+\int_{0}^{s} e^{\left(s-s^{\prime}\right) \mathcal{L}}(1-\mathcal{P}) \mathcal{N}\left(\phi\left(s^{\prime}\right)\right) \mathrm{d} s^{\prime}
$$

and thus,

$$
\begin{aligned}
\|(1-\mathcal{P}) \mathcal{K}(\phi, f)(s)\|_{X} & \lesssim e^{-\omega_{0} s}\|f\|_{X}+\int_{0}^{s} e^{-\omega_{0}\left(s-s^{\prime}\right)}\left\|\phi\left(s^{\prime}\right)\right\|_{X}^{2} \mathrm{~d} s^{\prime} \\
& \lesssim \frac{\delta}{c} e^{-\omega_{0} s}+\|\phi\|_{\mathcal{X}}^{2} e^{-\omega_{0} s} \int_{0}^{s} e^{-\omega_{0} s^{\prime}} \mathrm{d} s^{\prime} \\
& \lesssim \frac{\delta}{c} e^{-\omega_{0} s}+\delta^{2} e^{-\omega_{0} s}
\end{aligned}
$$

by Theorem 3.14 and Lemma 4.6. In summary, this yields $\|\mathcal{K}(\phi, f)\| \mathcal{X} \lesssim \frac{\delta}{c}+\delta^{2}$ and by choosing $c>0$ large enough and $\delta>0$ small enough, we obtain $\|\mathcal{K}(\phi, f)\| \mathcal{X} \leq \delta$.

Lemma 4.11 Let $\delta>0$ be sufficiently small. Then we have the bound

$$
\|\mathcal{K}(\phi, f)-\mathcal{K}(\psi, f)\| \mathcal{X} \leq \frac{1}{2}\|\phi-\psi\| \mathcal{X}
$$

for all $\phi, \psi \in \mathcal{X}_{\delta}$ and all $f \in X$.

Proof We have

$$
\mathcal{P K}(\phi, f)(s)-\mathcal{P} \mathcal{K}(\psi, f)(s)=-e^{s} \int_{s}^{\infty} e^{-s^{\prime}} \mathcal{P}\left[\mathcal{N}\left(\phi\left(s^{\prime}\right)\right)-\mathcal{N}\left(\psi\left(s^{\prime}\right)\right)\right] \mathrm{d} s^{\prime}
$$

and Lemma 4.6 yields

$$
\|\mathcal{P K}(\phi, f)(s)-\mathcal{P} \mathcal{K}(\psi, f)(s)\|_{X} \lesssim \delta e^{-2 \omega_{0} s}\|\phi-\psi\|_{\mathcal{X}},
$$

cf. the proof of Lemma 4.10. Similarly,

$(1-\mathcal{P}) \mathcal{K}(\phi, f)(s)-(1-\mathcal{P}) \mathcal{K}(\psi, f)(s)=\int_{0}^{s} e^{\left(s-s^{\prime}\right) \mathcal{L}}(1-\mathcal{P})\left[\mathcal{N}\left(\phi\left(s^{\prime}\right)\right)-\mathcal{N}\left(\psi\left(s^{\prime}\right)\right)\right] \mathrm{d} s^{\prime}$ and thus,

$$
\|(1-\mathcal{P}) \mathcal{K}(\phi, f)(s)-(1-\mathcal{P}) \mathcal{K}(\psi, f)(s)\|_{X} \lesssim \delta e^{-\omega_{0} s}\|\phi-\psi\|_{\mathcal{X}}
$$

In summary, we infer

$$
\|\mathcal{K}(\phi, f)-\mathcal{K}(\psi, f)\|_{\mathcal{X}} \lesssim \delta\|\phi-\psi\|_{\mathcal{X}}
$$

and by choosing $\delta>0$ sufficiently small, we arrive at the claim. 
Based on the above, it is now easy to construct a global solution to the modified equation

$$
\phi(s)=e^{s \mathcal{L}} \mathcal{U}(h, T)+\int_{0}^{s} e^{\left(s-s^{\prime}\right) \mathcal{L}} \mathcal{N}\left(\phi\left(s^{\prime}\right)\right) \mathrm{d} s^{\prime}-e^{s} \mathcal{C}(\phi, \mathcal{U}(h, T)) .
$$

Corollary 4.12 Let $M>0$ be sufficiently large and $\delta>0$ sufficiently small. Then, for every $h \in Y$ and every $T>0$ satisfying

$$
\|h\|_{Y}+|T-1| \leq \frac{\delta}{M},
$$

there exists a unique $\phi_{h, T} \in \mathcal{X}_{\delta}$ such that

$$
\phi_{h, T}=\mathcal{K}\left(\phi_{h, T}, \mathcal{U}(h, T)\right) .
$$

In particular, $\phi_{h, T}$ is a solution to Eq. (4.4). Furthermore, the solution map $(h, T) \mapsto \phi_{h, T}$ is continuous.

Proof By Lemma 4.9, we can achieve

$$
\|\mathcal{U}(h, T)\|_{X} \leq \frac{\delta}{c}
$$

for any given $c>0$ by choosing $M$ sufficiently large. Thus, the existence and uniqueness of $\phi_{h, T}$ is a consequence of Lemmas 4.10 and 4.11, and the contraction mapping principle.

For the continuity of the solution map we note that

$$
\begin{aligned}
\left\|\phi_{h_{1}, T_{1}}-\phi_{h_{2}, T_{2}}\right\|_{\mathcal{X}}= & \left\|\mathcal{K}\left(\phi_{h_{1}, T_{1}}, \mathcal{U}\left(h_{1}, T_{1}\right)\right)-\mathcal{K}\left(\phi_{h_{2}, T_{2}}, \mathcal{U}\left(h_{2}, T_{2}\right)\right)\right\|_{\mathcal{X}} \\
\leq & \left\|\mathcal{K}\left(\phi_{h_{1}, T_{1}}, \mathcal{U}\left(h_{1}, T_{1}\right)\right)-\mathcal{K}\left(\phi_{h_{2}, T_{2}}, \mathcal{U}\left(h_{1}, T_{1}\right)\right)\right\|_{\mathcal{X}} \\
& +\left\|\mathcal{K}\left(\phi_{h_{2}, T_{2}}, \mathcal{U}\left(h_{1}, T_{1}\right)\right)-\mathcal{K}\left(\phi_{h_{2}, T_{2}}, \mathcal{U}\left(h_{2}, T_{2}\right)\right)\right\|_{\mathcal{X}} \\
\leq & \frac{1}{2}\left\|\phi_{h_{1}, T_{1}}-\phi_{h_{2}, T_{2}}\right\| \mathcal{X}+C\left\|\mathcal{U}\left(h_{1}, T_{1}\right)-\mathcal{U}\left(h_{2}, T_{2}\right)\right\|_{X}
\end{aligned}
$$

by Lemma 4.11 since

$$
\begin{aligned}
& \left\|\mathcal{K}\left(\phi_{h_{2}, T_{2}}, \mathcal{U}\left(h_{1}, T_{1}\right)\right)(s)-\mathcal{K}\left(\phi_{h_{2}, T_{2}}, \mathcal{U}\left(h_{2}, T_{2}\right)\right)(s)\right\|_{X} \\
& \quad=\left\|e^{s \mathcal{L}}(1-\mathcal{P})\left[\mathcal{U}\left(h_{1}, T_{1}\right)-\mathcal{U}\left(h_{2}, T_{2}\right)\right]\right\|_{X} \\
& \quad \lesssim e^{-\omega_{0} s}\left\|\mathcal{U}\left(h_{1}, T_{1}\right)-\mathcal{U}\left(h_{2}, T_{2}\right)\right\|_{X}
\end{aligned}
$$

by Theorem 3.14. Consequently, Lemma 4.9 finishes the proof.

Corollary 4.12 provides us with a solution to the modified Eq. (4.4). Thus, in order to obtain a (mild) solution to Eq. (4.1), we have to get rid of the correction term $\mathcal{C}(\phi, \mathcal{U}(h, T))$. So far, $h$ and $T$ can be chosen freely, subject to the smallness conditions in Corollary 4.12. In the last step of the construction we now show that for any small $h \in Y$ there exists in fact a $T_{h}>0$ such that $\mathcal{C}\left(\phi_{h, T_{h}}, \mathcal{U}\left(h, T_{h}\right)\right)=0$.

Lemma 4.13 Let $M>0$ be sufficiently large and $\delta>0$ sufficiently small. Then, for every $h \in Y$ satisfying $\|h\|_{Y} \leq \frac{\delta}{M^{2}}$, there exists $a T_{h} \in\left[1-\frac{\delta}{M}, 1+\frac{\delta}{M}\right]$ such that

$$
\mathcal{C}\left(\phi_{h, T_{h}}, \mathcal{U}\left(h, T_{h}\right)\right)=0 .
$$

Proof For brevity we set $I_{M, \delta}=\left[1-\frac{\delta}{M}, 1+\frac{\delta}{M}\right]$. The map $\mathcal{C}$ has values in $\left\langle\psi_{1}\right\rangle$ and thus, it suffices to consider the real-valued function $F_{h}: I_{M, \delta} \rightarrow \mathbb{R}$ given by

$$
F_{h}(T):=\left(\mathcal{C}\left(\phi_{h, T}, \mathcal{U}(h, T)\right) \mid \psi_{1}\right)_{L_{\sigma}^{2}\left(\mathbb{R}^{5}\right)} .
$$


By Corollary $4.12, F_{h}$ is continuous. Furthermore, by noting that

$$
\left.\partial_{T} \frac{f_{0}(\sqrt{T}|x|)}{|x|}\right|_{T=1}=\frac{1}{2} f_{0}^{\prime}(|x|)=\frac{1}{2}\left\|f_{0}(|\cdot|)\right\|_{L_{\sigma}^{2}\left(\mathbb{R}^{5}\right)} \psi_{1}(x)
$$

we obtain by a Taylor expansion the representation

$$
\mathcal{U}(h, T)(x)=\frac{1}{2}(T-1)\left\|f_{0}(|\cdot|)\right\|_{L_{\sigma}^{2}\left(\mathbb{R}^{5}\right)} \psi_{1}(x)+(T-1)^{2} f_{T}(x)+h(\sqrt{T}|x|) /|x|
$$

where $T \mapsto f_{T}: I_{M, \delta} \rightarrow X$ is continuous and $\left\|f_{T}\right\|_{X} \lesssim 1$ for all $T \in I_{M, \delta}$. This yields

$$
\begin{aligned}
F_{h}(T) & =\left(\mathcal{C}\left(\phi_{h, T}, \mathcal{U}(h, T)\right) \mid \psi_{1}\right)_{L_{\sigma}^{2}\left(\mathbb{R}^{5}\right)} \\
& =\left(\mathcal{P U}(h, T) \mid \psi_{1}\right)_{L_{\sigma}^{2}\left(\mathbb{R}^{5}\right)}+\int_{0}^{\infty} e^{-s^{\prime}}\left(\mathcal{P} \mathcal{N}\left(\phi_{h, T}\left(s^{\prime}\right)\right) \mid \psi_{1}\right)_{L_{\sigma}^{2}\left(\mathbb{R}^{5}\right)} \mathrm{d} s^{\prime} \\
& =\frac{1}{2}(T-1)\left\|f_{0}(|\cdot|)\right\|_{L_{\sigma}^{2}\left(\mathbb{R}^{5}\right)}+O\left(\frac{\delta}{M^{2}} T^{0}\right)+O\left(\delta^{2} T^{0}\right) .
\end{aligned}
$$

Consequently, by setting $\tilde{F}_{h}(T)=2\left\|f_{0}(|\cdot|)\right\|_{L_{\sigma}^{2}\left(\mathbb{R}^{5}\right)}^{-1} F_{h}(T)$, we infer

$$
\tilde{F}_{h}(T)=T-1+O\left(\frac{\delta}{M^{2}} T^{0}\right)+O\left(\delta^{2} T^{0}\right)
$$

and $\tilde{F}_{h}(T)=0$ is equivalent to $T-1=G(T)$ for a continuous function $G: I_{M, \delta} \rightarrow \mathbb{R}$ that satisfies

$$
|G(T)| \leq C \frac{\delta}{M^{2}}+C \delta^{2}
$$

for all $T \in I_{M, \delta}$. By choosing $M>0$ sufficiently large and $\delta>0$ sufficiently small, we can achieve $|G(T)| \leq \frac{\delta}{M}$ for all $T \in I_{M, \delta}$ and thus, $1+G$ is a continuous self-map of the interval $I_{M, \delta}$ which necessarily has a fixed point $T_{h} \in I_{M, \delta}$.

\subsection{Proof of Theorem 1.2}

Without loss of generality we set $T_{0}=1$. Lemma 4.13 yields a strong solution $w_{h}(y, s)=$ $\phi_{h, T_{h}}(s)\left(y e_{1}\right)$ of Eq. (2.5) with $T=T_{h}$ and

$$
\left\|w_{h}(|\cdot|, s)\right\|_{X} \leq \delta e^{-\omega_{0} s} .
$$

By construction, see Sect. 2,

$$
u_{h}(r, t):=f_{0}\left(\frac{r}{\sqrt{T_{h}-t}}\right)+\frac{r}{\sqrt{T_{h}-t}} w_{h}\left(\frac{r}{\sqrt{T_{h}-t}},-\log \left(T_{h}-t\right)+\log T_{h}\right)
$$

is a solution of Eq. (1.1) with initial data $u_{h}(r, 0)=u_{1}^{*}(0, r)+h(r)$. By scaling, we infer

$$
\begin{aligned}
\left\|u_{h}(\cdot, t)-u_{T_{h}}^{*}(\cdot, t)\right\|_{Y} & =\left(T_{h}-t\right)^{-\frac{1}{2}}\left\|w_{h}\left(\frac{|\cdot|}{\sqrt{T_{h}-t}},-\log \left(T_{h}-t\right)+\log T_{h}\right)\right\|_{X} \\
& \lesssim\left(T_{h}-t\right)^{-\frac{5}{4}}\left\|w_{h}\left(|\cdot|,-\log \left(T_{h}-t\right)+\log T_{h}\right)\right\|_{X} \\
& \lesssim \delta\left(T_{h}-t\right)^{-\frac{5}{4}+\omega_{0}}
\end{aligned}
$$

for all $t \in\left[0, T_{h}\right)$. Furthermore, from Corollary 4.8 it follows that $f_{0}$ belongs to $Y$ and the blowup speed of $u_{T_{h}}^{*}$ in $Y$ is 


$$
\begin{aligned}
\left\|u_{T_{h}}^{*}(\cdot, t)\right\|_{Y} & =\left\||\cdot|^{-1} f_{0}\left(\frac{|\cdot|}{\sqrt{T_{h}-t}}\right)\right\|_{X} \\
& =\left(T_{h}-t\right)^{-\frac{1}{4}}\left\||\cdot|^{-1} f_{0}(|\cdot|)\right\|_{\dot{H}^{2}\left(\mathbb{R}^{5}\right)}+\left(T_{h}-t\right)^{-\frac{5}{4}}\left\||\cdot|^{-1} f_{0}(|\cdot|)\right\|_{\dot{H}^{4}\left(\mathbb{R}^{5}\right)} \\
& \simeq\left(T_{h}-t\right)^{-\frac{5}{4}}
\end{aligned}
$$

Consequently, the statement of Theorem 1.2 follows by choosing $M$ sufficiently large.

Acknowledgements Open access funding provided by University of Vienna.

\section{Compliance with ethical standards}

Conflict of interest The authors declare that they have no conflict of interest.

Open Access This article is distributed under the terms of the Creative Commons Attribution 4.0 International License (http://creativecommons.org/licenses/by/4.0/), which permits unrestricted use, distribution, and reproduction in any medium, provided you give appropriate credit to the original author(s) and the source, provide a link to the Creative Commons license, and indicate if changes were made.

\section{References}

1. Biernat, P.: Non-self-similar blow-up in the heat flow for harmonic maps in higher dimensions. Nonlinearity 28(1), 167-185 (2015)

2. Biernat, P., Bizoń, P.: Shrinkers, expanders, and the unique continuation beyond generic blowup in the heat flow for harmonic maps between spheres. Nonlinearity 24(8), 2211-2228 (2011)

3. Biernat, P., Donninger, R.: Construction of a spectrally stable self-similar blowup solution to the supercritical harmonic map heat flow. Preprint arXiv:1610.09496 (2016)

4. Biernat, P., Seki, Y.: Type II blow-up mechanism for supercritical harmonic map heat flow. Preprint arXiv:1601.01831 (2016)

5. Bizoń, P., Wasserman, A.: Nonexistence of shrinkers for the harmonic map flow in higher dimensions. Int. Math. Res. Not. IMRN 17, 7757-7762 (2015)

6. Bricmont, J., Kupiainen, A.: Universality in blow-up for nonlinear heat equations. Nonlinearity 7(2), 539-575 (1994)

7. Chang, K.-C., Ding, W.Y., Ye, R.: Finite-time blow-up of the heat flow of harmonic maps from surfaces. J. Differ. Geom. 36(2), 507-515 (1992)

8. Chen, Y.M., Ding, W.Y.: Blow-up and global existence for heat flows of harmonic maps. Invent. Math. 99(3), 567-578 (1990)

9. Collot, C., Raphaël, P., Szeftel, J.: On the stability of type I blow up for the energy super critical heat equation. Preprint arXiv:1605.07337 (2016)

10. Coron, J.-M., Ghidaglia, J.-M.: Explosion en temps fini pour le flot des applications harmoniques. C. R. Acad. Sci. Paris Sér. I Math. 308(12), 339-344 (1989)

11. Donninger, R.: On stable self-similar blowup for equivariant wave maps. Commun. Pure Appl. Math. 64(8), 1095-1147 (2011)

12. Donninger, R.: Stable self-similar blowup in energy supercritical Yang-Mills theory. Math. Z. 278(3-4), 1005-1032 (2014)

13. Donninger, R., Schörkhuber, B.: Stable self-similar blow up for energy subcritical wave equations. Dyn. Partial Differ. Equ. 9(1), 63-87 (2012)

14. Donninger, R., Schörkhuber, B.: Stable blow up dynamics for energy supercritical wave equations. Trans. Am. Math. Soc. 366(4), 2167-2189 (2014)

15. Donninger, R., Schörkhuber, B.: Stable blowup for wave equations in odd space dimensions. Preprint arXiv: 1504.00808 (2015)

16. Donninger, R., Schörkhuber, B.: On blowup in supercritical wave equations. Commun. Math. Phys. 346(3), 907-943 (2016)

17. Donninger, R., Schörkhuber, B.: Stable blowup for the supercritical Yang-Mills heat flow. Preprint arXiv: 1604.07737 (2016)

18. Eells, J., Lemaire, L.: Two Reports on Harmonic Maps. World Scientific Publishing Co. Inc, River Edge (1995) 
19. Eells, J., Ratto, A.: Harmonic Maps and Minimal Immersions with Symmetries: Methods of Ordinary Differential Equations Applied to Elliptic Variational Problems. Volume 130 of Annals of Mathematics Studies. Princeton University Press, Princeton (1993)

20. Fan, H.: Existence of the self-similar solutions in the heat flow of harmonic maps. Sci. China Ser. A 42(2), 113-132 (1999)

21. Gastel, A.: Singularities of first kind in the harmonic map and Yang-Mills heat flows. Math. Z. 242(1), 47-62 (2002)

22. Germain, P., Ghoul, T.-E., Miura, H.: On uniqueness for the harmonic map heat flow in supercritical dimensions. Preprint arXiv:1601.06601 (2016)

23. Germain, P., Rupflin, M.: Selfsimilar expanders of the harmonic map flow. Ann. Inst. H. Poincaré Anal. Non Linéaire 28(5), 743-773 (2011)

24. Giga, Y., Kohn, R.V.: Asymptotically self-similar blow-up of semilinear heat equations. Commun. Pure Appl. Math. 38(3), 297-319 (1985)

25. Guan, M., Gustafson, S., Tsai, T.-P.: Global existence and blow-up for harmonic map heat flow. J. Differ. Equ. 246(1), 1-20 (2009)

26. Hélein, F.: Harmonic Maps, Conservation Laws and Moving Frames, Volume 150 of Cambridge Tracts in Mathematics, 2nd edn. Cambridge University Press, Cambridge (2002). Translated from the 1996 French original, With a foreword by James Eells

27. Jr James, E., Sampson, J.H.: Harmonic mappings of Riemannian manifolds. Am. J. Math. 86, 109-160 (1964)

28. Lin, F., Wang, C.: The Analysis of Harmonic Maps and Their Heat Flows. World Scientific Publishing Co. Pte. Ltd., Hackensack (2008)

29. Merle, F., Zaag, H.: Stability of the blow-up profile for equations of the type $u_{t}=\Delta u+|u|^{p-1} u$. Duke Math. J. 86(1), 143-195 (1997)

30. Qing, J.: On singularities of the heat flow for harmonic maps from surfaces into spheres. Commun. Anal. Geom. 3(1-2), 297-315 (1995)

31. Qing, J., Tian, G.: Bubbling of the heat flows for harmonic maps from surfaces. Commun. Pure Appl. Math. 50(4), 295-310 (1997)

32. Raphaël, P., Schweyer, R.: Stable blowup dynamics for the 1-corotational energy critical harmonic heat flow. Commun. Pure Appl. Math. 66(3), 414-480 (2013)

33. Raphaël, P., Schweyer, R.: Quantized slow blow-up dynamics for the corotational energy-critical harmonic heat flow. Anal. PDE 7(8), 1713-1805 (2014)

34. Sacks, J., Uhlenbeck, K.: The existence of minimal immersions of 2-spheres. Ann. Math. (2) 113(1), 1-24 (1981)

35. Schoen, R., Yau, S.T.: Lectures on harmonic maps. In: Conference Proceedings and Lecture Notes in Geometry and Topology, II. International Press, Cambridge (1997)

36. Smith, R.T.: Harmonic mappings of spheres. Am. J. Math. 97, 364-385 (1975)

37. Struwe, M.: On the evolution of harmonic mappings of Riemannian surfaces. Comment. Math. Helv. 60(4), 558-581 (1985)

38. Struwe, M.: On the evolution of harmonic maps in higher dimensions. J. Differ. Geom. 28(3), 485-502 (1988)

39. Struwe, M.: The evolution of harmonic maps: existence, partial regularity, and singularities. In: Lloyd, N.G., Ni, W.M., Peletier, L.A., Serrin, J. (eds.). Proceedings of the conference held in Gregynog, August 20-29, 1989. Nonlinear Diffusion Equations and Their Equilibrium States, 3. Progress in Nonlinear Differential Equations and Their Applications, vol 7. Birkhäuser, Boston, MA

40. Tayachi, S., Zaag, H.: Existence of a stable blow-up profile for the nonlinear heat equation with a critical power nonlinear gradient term. Preprint arXiv:1506.08306 (2015)

41. Taylor, M.E.: Partial Differential Equations III. Nonlinear Equations, Volume 117 of Applied Mathematical Sciences, 2nd edn. Springer, New York (2011)

42. Teschl, G.: Mathematical Methods in Quantum Mechanics: With Applications to Schrödinger Operators. Volume 157 of Graduate Studies in Mathematics, 2nd edn. American Mathematical Society, Providence (2014)

43. Topping, P.: Winding behaviour of finite-time singularities of the harmonic map heat flow. Math. Z. 247(2), 279-302 (2004)

44. van den Berg, J.B., Hulshof, J., King, J.R.: Formal asymptotics of bubbling in the harmonic map heat flow. SIAM J. Appl. Math 63(5), 1682-1717 (2003). (electronic)

45. Whitney, H.: Differentiable even functions. Duke Math. J. 10, 159-160 (1943) 